\title{
Anthropogenic infilling of a Bermudian sinkhole and its impact on sedimentation and benthic foraminifera in the adjacent anchialine cave environment
} \author{
and Gil Nolan ${ }^{5}$ \\ ${ }^{1}$ Department of Marine Biology, Texas A\&M University at Galveston, Galveston, Texas, 77554, USA \\ ${ }^{2}$ Department of Marine Sciences, Texas A\&M University at Galveston, Galveston, Texas, 77554, USA \\ ${ }^{3}$ Department of Oceanography, Texas A\&M University, College Station, Texas, 77843, USA \\ ${ }^{4}$ Bermuda Institute of Ocean Sciences, Ferry Reach, St George's Parish, GE 01, Bermuda \\ ${ }^{5}$ Bermuda Zoological Society, Flatts, Hamilton Parish, FL BX, Bermuda
}

Jacquelyn N. Cresswell ${ }^{1}$, Peter J. van Hengstum ${ }^{2,3^{*}}$, Thomas M. Iliffe ${ }^{1}$, Bruce E. Williams ${ }^{4}$,

\begin{abstract}
In the mid-20 $20^{\text {th }}$ century, an inland brackish pond from Bermuda, known as Eve's Pond, was filled with marine sediment from an adjacent coastal lagoon. At this time, an eyewitness reported "...sediment billowing out of the Green Bay Cave for days...", which is a marinedominated anchialine cave located proximal to the former location of Eve's Pond ( 200 m). The purpose of this study was to evaluate the potential impact of this infilling event on cave sedimentation and benthic meiofaunal communities, as proxied by the unicellular protists foraminifera that remain preserved in the sediment record. Eight sediment cores were collected from an underwater passage in Green Bay Cave in a transect towards the location where Eve's Pond was surveyed in 1901 CE. The sediment cores were analyzed for visual and density changes (photography, X-radiography), textural variability, benthic foraminifera fauna and diversity, and radiocarbon dating. The recovered sediment cores mostly sampled a late Holocene carbonate mud facies that had been described during previous research in the cave, with benthic foraminiferal assemblages post-dating the onset of seawater circulating between the saline groundwater flooding the cave and the adjacent Harrington Sound $\sim 1,900$ years ago. However, two cores located further into the cave (cores 13 and 17) contain a carbonate sand layer with lagoon foraminifera that is anomalous with respect to the Holocene depositional history of the cave and is most likely related to the mid-20 th century infilling of Eve's Pond. Examination of these two cores showed that after the infilling event, the community of benthic foraminifera rapidly reverted to pre-impact assemblages with foraminiferal stygophiles (e.g., Spirophthalmidium emaciatum, Sigmoilina tenuis), which were not displaced by new colonizers introduced into the cave by the dredge spoils. We caution that the results cannot be extrapolated to the pelagic crustacean community, but the results suggest that this physical sedimentary disturbance only minimally impacted the benthic foraminifera community in the cave passages that were sampled.
\end{abstract}

Keywords: Bermuda, anchialine, submarine, foraminifera, karst

Received 10 April 2017; Revised 17 August 2017; Accepted 17 August 2017

Citation: $\quad$ Cresswell J.N., van Hengstum P.J., lliffe T.M., Williams B.E. and Nolan G., 2017. Anthropogenic infilling of a Bermudian sinkhole and its impact on sedimentation and benthic foraminifera in the adjacent anchialine cave environment. International Journal of Speleology, 46 (3), 409-425. Tampa, FL (USA) ISSN 0392-6672 https://doi.org/10.5038/1827-806X.46.3.2128

\section{INTRODUCTION}

Anchialine and submarine cave environments globally host unique ecosystems with a high biodiversity of endemic fauna (Iliffe et al., 1983; Iliffe et al., 1984; Hayami, 1993; Miller, 1996; Sarbu et al., 1996), and it remains relatively unknown how these ecosystems will be impacted by anthropogenic activities in the $21^{\text {st }}$ century. Preliminary observations suggest that increasing sea surface temperatures from climate change may negatively impact anchialine ecosystems (Chevaldonné \& Lejeusne, 2003; Parravicini et al., 2010; Moritsch et al., 2014). Anthropogenic contaminants have also been measured in subterranean aquifers (Iliffe et al., 1984; Metcalfe et al., 2011), and the impact of these contaminants on metabolic pathways in anchialine "living fossil" species remains poorly understood 
(Kløve et al., 2014). It has also been hypothesized that coastal urbanization will promote habitat loss in anchialine environments (Iliffe et al., 1984; Gibbons, 2003; Nevill et al., 2010). Additional information on how anchialine environments have responded to previous environmental stressors may help illuminate how they will change during $21^{\text {st }}$ century environmental pressures (e.g., coastal urbanization, marine climate change).

Some coastal underwater caves have significant Holocene sediment accumulations that can preserve records of environmental change. Indeed, not all submerged caves have experienced sedimentation during the Holocene (Fornós et al., 2014). This is because underwater caves are significantly influenced by the point source effect (van Hengstum et al., 2015a) in which cave sedimentation is dependent on sedimentary particles transported into the cave from adjacent terrestrial and aquatic environments through karst openings (e.g., Carwash Cave: van Hengstum et al. (2010); Green Bay Cave: van Hengstum et al. (2011), and Yax Chen Cave: Collins et al. (2015)). However, once caves become inundated with seawater that is well-circulated with the ocean, the potential for autochthonous sedimentation from both biologic and inorganic sources can improve, but sedimentation throughout flooded caves remains dependent on factors such as local hydrodynamics, biologic activity, and sedimentary processes in adjacent coastal environments (Radolović et al., 2015). Despite the complexities of sedimentation in underwater caves, cave sediments can preserve long-term records of environmental change, both inside (van Hengstum et al., 2010; Collins et al., 2015) and outside the cave system (Kitamura et al., 2007; van Hengstum et al., 2010, 2015).

Cave sediment can also preserve microfossils that are useful proxies of long-term aquatic environmental change, such as bivalves (Kitamura et al., 2007), gastropods (Moolenbeek et al., 1989; Kase \& Hayami, 1992; Kano \& Kase, 2008), ostracodes (Maddocks \& Iliffe, 1986; Kornicker et al., 1998), benthic foraminifera (Javaux \& Scott, 2003; van Hengstum \& Scott, 2011, 2012), and testate amoeba (van Hengstum et al., 2009a). Given their statistically significant and diverse populations in small sediment samples, benthic foraminifera are particularly useful microfossils for environmental monitoring in coastal environments (Gooday et al., 1992; Gupta \& Machain-Castillo, 1993; Murray, 2001). Benthic foraminifera are singlecelled protists, the majority of which secrete a calcium carbonate or agglutinated shell or test that remains in the sediment long after the organism has died (Gooday et al., 1992; Lea et al., 2003). Benthic foraminifera readily colonize most marine habitats, form discrete assemblages in different coastal environments (e.g., reefs, lagoons, marshes), and rapidly respond to changes in salinity, temperature, and organic matter supply (Boltovskoy et al., 1991; Murray, 2001; Martin et al., 2002; Waelbroeck et al., 2002; Dissard et al., 2010). As such, subfossil benthic foraminifera in the stratigraphic record are widely used to reconstruct marine environmental change (Murray, 2001), and have been recently used to reconstruct anchialine and submarine cave environments (van Hengstum et al., 2009b, 2010; van Hengstum \& Scott, 2012).

In the mid-20th century, Eve's Pond in Bermuda was backfilled with marine sediment sourced from an adjacent lagoon (i.e., Flatt's Inlet). Recently, there have been local-scale discussions about re-excavating the pond to its original dimensions to return the landscape to its original ecology. However, it remains speculative as to (i) whether Eve's Pond was originally connected to Green Bay Cave through a conduit opening, (ii) the potential impact the mid-20th century infilling event had on the underwater cave habitats, and (iii) the potential impacts of re-connecting the cave to an adjacent pond. The objectives of this study are to analyze the stratigraphy and benthic foraminifera in sediment cores collected from submerged cave passages adjacent to the purported location of Eve's Pond to understand the original connectivity between the pond and Green Bay Cave, and to evaluate whether the infilling event impacted the cave's benthic foraminifera.

\section{STUDY SITE}

The North Atlantic island of Bermuda (Fig. 1A) is generally considered to be a Cover Collapse Island according to the Carbonate Island Karst Model of Mylroie and Mylroie (2007) because of its Eoceneaged basaltic core that is capped by alternating Quaternary paleosols and carbonates (Vacher, 1988). These Quaternary carbonates have since weathered into a matured karst landscape, most especially in the oldest limestone formations (Land et al., 1967; Mylroie et al., 1995; Mylroie \& Mylroie, 2007). Green Bay Cave is a large underwater cave in Bermuda with more than two kilometers of underwater passages. There are currently two primary entrances into the system: a subaerial sinkhole-based entrance (Cliff Pool Sinkhole) and a submarine entrance at the end of Green Bay, part of the restricted Harrington Sound. Hydrographically, Green Bay Cave is currently flooded by well-oxygenated saline groundwater that is tidally circulated through the entrance in Green Bay lagoon. A local brackish meteoric lens can be observed only in a few locations (e.g., Cliff Pool Sinkhole, Letter Box, and Air Dome), and can completely disappear during prolonged drought. Green Bay Cave is also the international type locality for several endemic cave crustaceans to satisfy rules according to the International Code of Zoological Nomenclature, including Mictocaris halope (Bowman \& Iliffe, 1985), Procaris chacei (Hart \& Manning, 1986), and Spelaeoecia bermudensis (Angel \& Iliffe, 1987).

Previous research has documented that environmental conditions in Green Bay Cave over the last 8000 years have been driven by Holocene sealevel rise in the North Atlantic Ocean. Prior to the Holocene inundation, Green Bay Cave was in the vadose zone and air-filled when sea levels were lower during maximum extant of global ice sheets during the Last Glacial Maximum. However, by 8,000 Cal yrs $\mathrm{BP}$ (a minimum age, new IntCal13 calibration results 


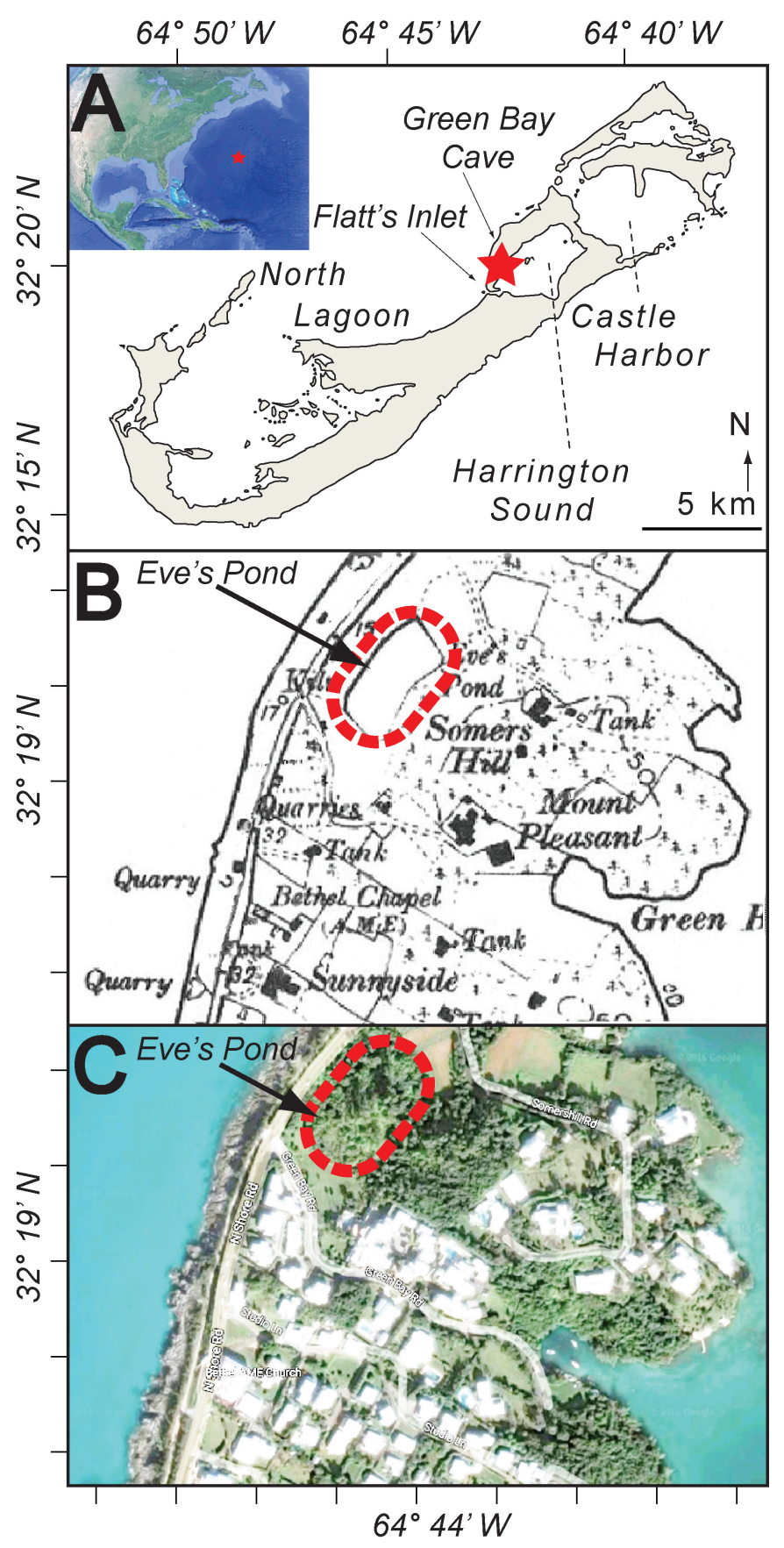

Fig. 1. A) Study site on the main island of Bermuda with inset depicting location in the North Atlantic Ocean; B) Original survey of Lieutenant A.J. Savage depicting the location of Eve's Pond on narrow strip of land separating Harrington Sound and North Shore Lagoon (Savage, 1901); C) Present day Eve's Pond location.

described in the Methods section), concomitant sealevel and groundwater-level rise flooded the floor of Green Bay Cave to create an aquatic cave environment, and by $\sim 7,600 \mathrm{Cal}$ yrs BP, the water table had risen to the elevation of the cave ceiling (van Hengstum et al., 2011). From $\sim 7,600$ to 1,900 Cal yrs BP, episodic sedimentation was dominated by particles transported into the cave from the adjacent terrestrial surface (van Hengstum et al., 2011). Subfossil benthic foraminifera at this time indicate that Green Bay Cave was first flooded by dysoxic (0.1-0.3 ml/1), saline groundwater (van Hengstum \& Scott, 2012), similar to anchialine environments on the Yucatan Peninsula in Mexico. Lastly, a cave-wide initiation of carbonate mud deposition occurred at $\sim 1,900 \mathrm{Cal}$ yrs BP, which is thought related to inundation of
Flatts Inlet, and onset of modern circulation between Harrington Sound, North Shore Lagoon, and Green Bay Cave. Based on subfossil assemblages of benthic foraminifera, it is likely that Green Bay Cave was initially flooded by saline groundwater with low oxic dissolved oxygen concentrations (1.5-3 ml/1), which indicates that saline groundwater flooding the cave was initially poorly circulated with seawater from outside the cave (van Hengstum \& Scott, 2012). Over the last millennium, however, benthic foraminifera significantly diversified as the cave benthos gradually became more oxygenated from increased circulation of seawater between Harrington Sound and North Shore Lagoon/Green Bay Cave through tidal pumping (van Hengstum \& Scott, 2012).

In 1901 CE, British Lieutenant A.J. Savage surveyed and mapped an inland pond on the north shore of Harrington Sound that he labeled Eve's Pond (Savage, 1901) (Fig. 1B). Later, in the mid-20th century, Eve's Pond was allegedly filled with marine sediment dredged from an adjacent lagoon-purportedly Flatt's Inlet. Following this infilling event, eyewitnesses reported white sediment flowing out of the marine entrance to Green Bay Cave for days. Given the proximity of submerged passages in Green Bay Cave to the recorded position of Eve's Pond on the early topographic map of Bermuda (Savage, 1901), it is speculated that Green Bay Cave was once connected to an opening in Eve's Pond. Based on a cave survey completed in the late 1980's CE by Mr. Robert Power, the tunnel leading from the submarine entrance at Green Bay to the Letter Box area would be a potential passage that could have led to the former Eve's Pond. However, there is an unmapped tunnel near the base of the Letter Box, which may also have served as a conduit of dredge spoils from Eve's Pond through Green Bay Cave.

We are motivated by the following questions: (a) was Eve's Pond originally interconnected into the Green Bay, (b) if so, did the infilling of Eve's Pond leave a sedimentary deposit in the stratigraphic record, and (c) what was the impact of the infilling event on the benthic foraminiferal populations? The study area for the current research was the passage extending from the submarine entrance at Green Bay, to a large room that continues into an area called the Letter Box (Fig. 2). Today, this passage is primarily located at $\sim 15 \mathrm{~m}$ below sea level, but contains two prominent boulder breakdown piles between open water and the Letter Box (Fig. 2). Particles at the sediment-water interface are primarily silt-sized, but sediment texture coarsens to sand particles towards the submarine cave entrance at Green Bay (van Hengstum et al., 2011).

\section{METHODS}

Sediment push cores were collected by divers using advanced technical cave diving procedures, with divers meeting or exceeding safety standards established by the American Academy of Underwater Sciences. Before coring, collapsible fiberglass avalanche rulers were used to probe the subsurface stratigraphy for 


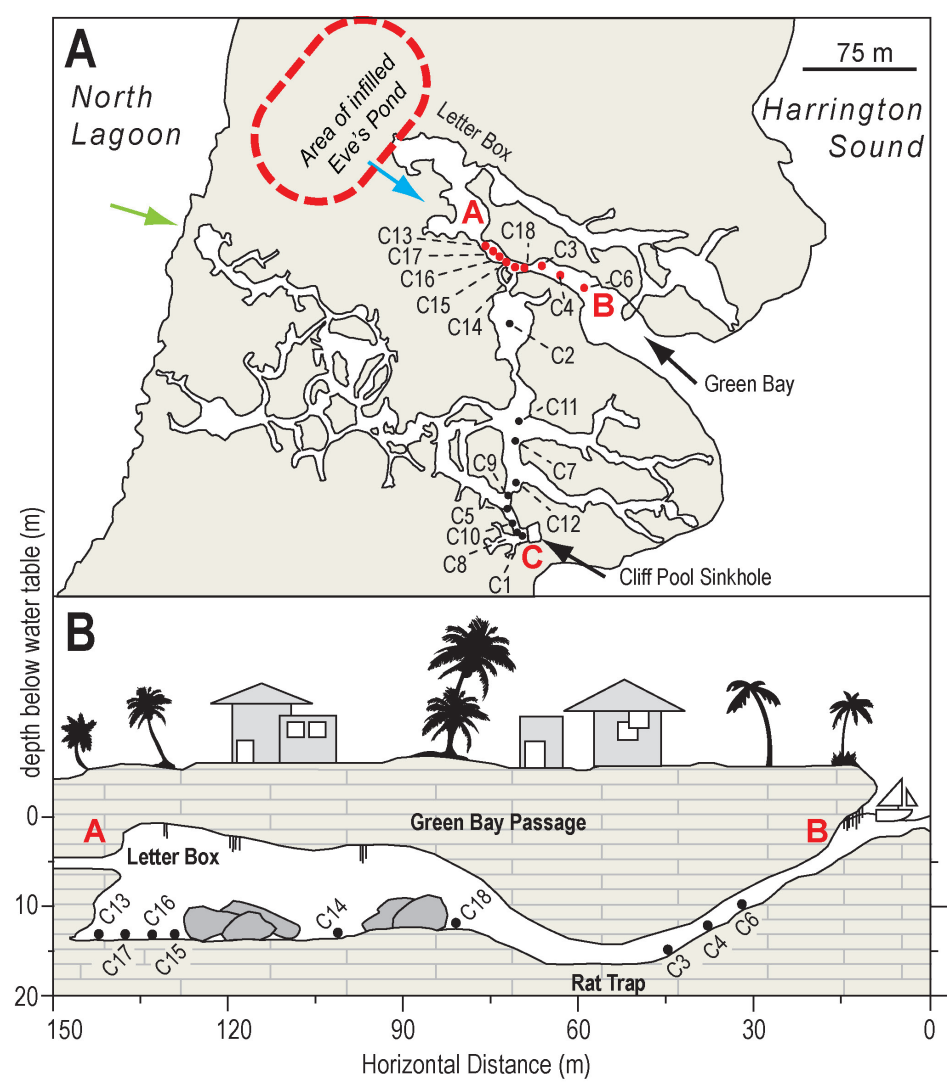

Fig. 2. A) Detailed map of Green Bay Cave with locations of sediment cores 1 through 12 (van Hengstum et al., 2011) and 13 through 18 (this study). Green arrow represents suspected now-collapsed karst window based on foraminiferal evidence (van Hengstum \& Scott, 2011), black arrows represent current entrances at Green Bay Lagoon and Cliff Pool Sinkhole and blue arrow represents area where some former passage connected into Eve's Pond; B) Profile view of Green Bay Cave from A to B with core locations.

sediment depth and texture in over 50 localities in the study area. The sediment probing revealed a clearly defined, but spatially discontinuous, coarse grained sedimentary deposit in the shallow subsurface in cave areas most proximal to the Letter Box. Further coring efforts attempted to sample this discontinuous deposit and characterize its lateral continuity and variability. Eight sediment cores were collected in a transect through the Green Bay Passage, from the Letter Box towards the submarine cave entrance in Green Bay (Fig. 2).

Textural variability in the cores was examined in incremental $1 \mathrm{~cm}$ sediment intervals downcore with: (i) standard loss on ignition procedures (at $550^{\circ} \mathrm{C}$ for $4.5 \mathrm{hrs}$ ) to estimate bulk organic matter (Heiri et al., 2001) and (ii) downcore particle size distributions and statistics (e.g., mean, mode, and standard deviation) measured with a Malvern Mastersizer 2000S laser particle size analyzer (Sperazza et al., 2004). Sediment cores were dominated by carbonate particles that were easily disaggregated in sodium hexametaphosphate dispersant, so no chemical digestions were completed prior to laser particle size determination (e.g., $\mathrm{HCl}, \mathrm{H}_{2} \mathrm{O}_{2}$ ).

Chronological constraint for the cores was established with a stratigraphic comparison to previously analyzed sediment cores from Green Bay Cave (van Hengstum et al., 2011), and radiocarbon dating of an additional marine bivalve (e.g., Barbatia domingensis). No terrestrial plant macrofossils were recovered in the sediment cores, necessitating the use of carbonate material. The bivalve Barbatia domingensis is a short-lived and sessile mollusk that is abundant in Bermuda's submarine caves, and detaches from the cave walls upon death to become part of the sediment record (van Hengstum $\&$ Scott, 2011). One B. domingensis was collected from Core 13 and sent to National Ocean Sciences Accelerator Mass Spectrometry facility at Woods Hole Oceanographic Institution for radiocarbon analysis (Table 1). Recent radiocarbon analysis of marine bivalves, gastropods, ostracodes and foraminifera collected from the sediment-water interface Cow Cave and Walsingham Cave in Bermuda (van Hengstum, unpublished data) indicate that the marine reservoir collection that was originally applied by van Hengstum et al. (2011) is not necessarily required for biogenic carbonates from Bermuda caves. As such, newly obtained conventional radiocarbon dates for this study, and those previously published from van Hengstum et al. (2011), were all calibrated into calendar years before present using only IntCal13 in the freeware program Calib 7.1 (Reimer et al., 2013).

Core 13 (GB-C13) was selected for detailed benthic foraminiferal analysis because it contained the most expanded sedimentary record. Benthic foraminifera were first concentrated by wet sieving $1.25 \mathrm{~cm}^{3}$ or $2.5 \mathrm{~cm}^{3}$ of bulk sediment samples over standard 45-500 $\mu \mathrm{m}$ screen meshes, with the remaining coarse sediment residues split using a wet splitter (Scott \& Hermelin, 1993) to enable representative census counts of $\sim 300$ individuals per sample. Individual benthic foraminifera were wet picked onto micropaleontologic slides and enumerated into an original data matrix of 32 samples $\times 123$ observations, with taxonomy confirmed by scanning electron microscopy of representative individuals and literature comparisons (Carman, 1933; Bermúdez, 1949; Loeblich Jr \& Tappan, 1987; Javaux \& Scott, 2003; van Hengstum \& Scott, 2011). The final data matrix of raw relative abundance data was log transformed to emphasize broader community patterns (Legendre \& Legendre, 1998), and then exposed to stratigraphically-constrained Q-mode cluster analysis using a Euclidian distance coefficient to identify biofacies downcore. As a test of reproducibility, similar groupings appeared in the dendrograms produced from Q-mode cluster analysis using other distance coefficients. Lastly, species richness (R) and Shannon-Wiener Diversity Index $(H)$ were calculated using Paleontological Statistics (PAST). Discussion of dissolved oxygen in seawater follow Kaiho (1994): high oxic (>3 ml/1), low oxic (3-1.5 ml/1), suboxic (0.3-1.5), dysoxic (0.1-0.3 ml/1), and anoxic $(<0.1 \mathrm{ml} / 1)$.

\section{RESULTS}

\section{Sedimentology and chronology}

The previous stratigraphic work in Green Bay Cave documented a cave-wide shift to carbonate deposition at $\sim 2,000$ Cal yrs BP (Fig. 3). Elsewhere, Vollbrecht (1996) completed an extensive stratigraphic, 


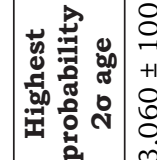

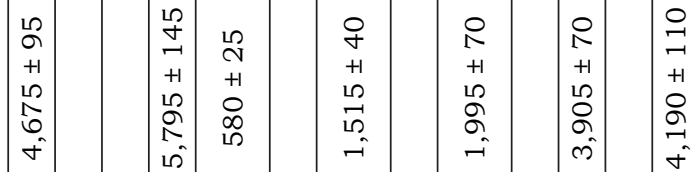

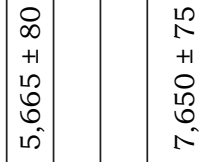

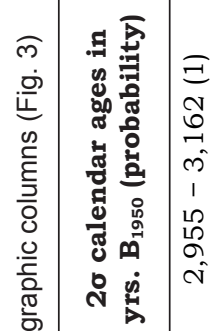

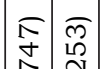

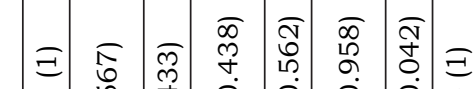

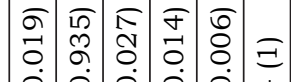

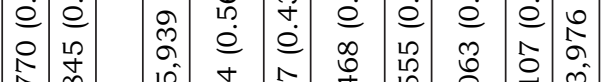

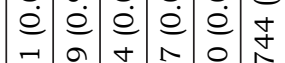

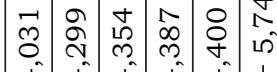

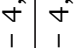

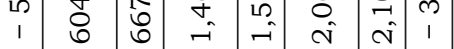

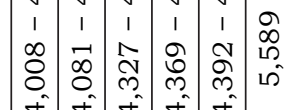

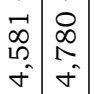

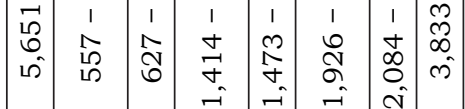

离画

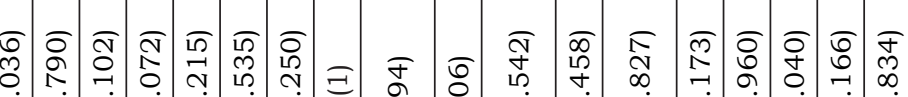

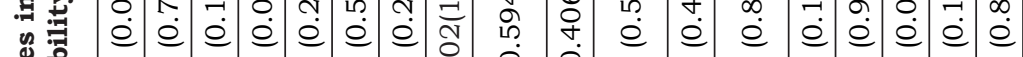

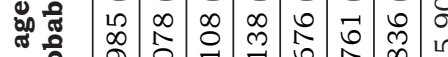

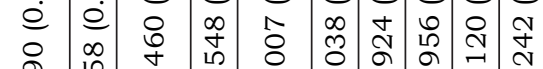

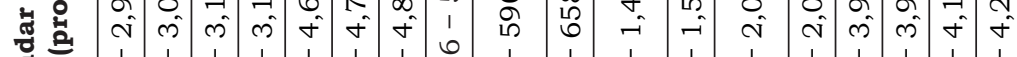

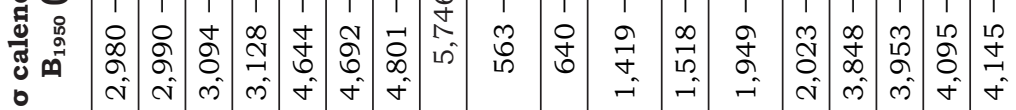

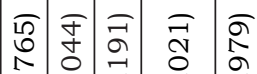

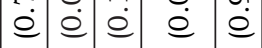

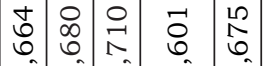

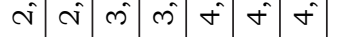

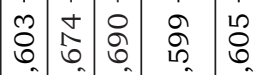

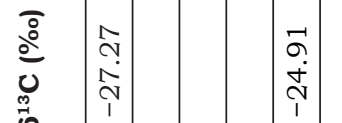

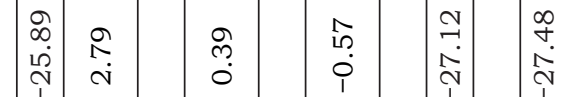

ம०

政

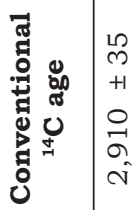

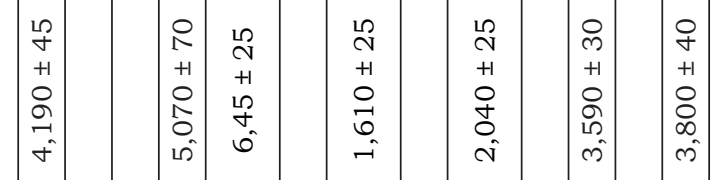

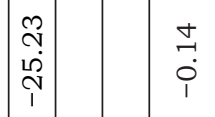

\begin{tabular}{|c|c|c|c|c|c|c|c|c|c|c|}
\hline $\begin{array}{l}0 \\
0 \\
\vdots\end{array}$ & $\mid \begin{array}{c}0 \\
\tilde{O} \\
0 \\
0 \\
+1 \\
+1 \\
0 \\
0 \\
0 \\
0\end{array}$ & $\begin{array}{l}0 \\
\tilde{O} \\
0 \\
0 \\
0 \\
+1 \\
\hat{1} \\
\hat{\infty} \\
0 \\
\hat{ம} \\
0\end{array}$ & 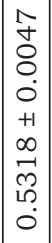 & 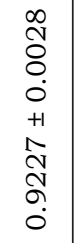 & 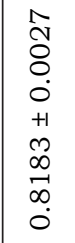 & $\begin{array}{l}\stackrel{+}{N} \\
\stackrel{0}{0} \\
\dot{0} \\
+1 \\
\hat{N} \\
\stackrel{R}{N} \\
\stackrel{0}{0}\end{array}$ & $\begin{array}{l}2 \\
\hat{N} \\
0 \\
0 \\
0 \\
+1 \\
+1 \\
\hat{\alpha} \\
\tilde{B} \\
0 \\
0\end{array}$ & $\begin{array}{l}\mathcal{N} \\
\tilde{O} \\
0 \\
0 \\
0 \\
+1 \\
0 \\
0 \\
\hat{N} \\
0 \\
0 \\
0\end{array}$ & $\begin{array}{l}\text { N } \\
\tilde{O} \\
0 \\
0 \\
0 \\
+1 \\
ت \\
\vec{y} \\
1 \\
0 \\
0\end{array}$ & 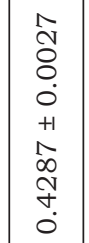 \\
\hline 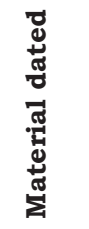 & $\mid$\begin{tabular}{|c|}
0.0 \\
.00 \\
3 \\
3
\end{tabular} & $\begin{array}{l}-\infty \\
-50 \\
3 \\
3\end{array}$ & $\begin{array}{c}0 \\
.00 \\
\overrightarrow{3} \\
-\end{array}$ & 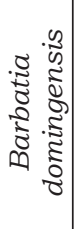 & 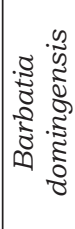 & 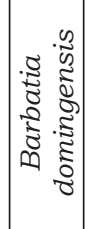 & 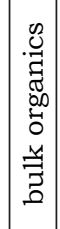 & 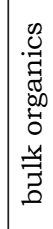 & 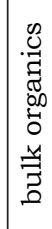 & 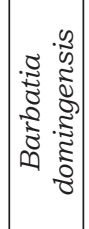 \\
\hline 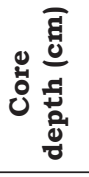 & $\stackrel{-}{\sim}$ & 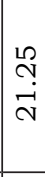 & $\begin{array}{l}n \\
i \\
i\end{array}$ & 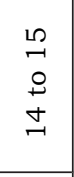 & 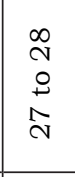 & $\begin{array}{l}n \\
\dot{1} \\
m \\
0 \\
0 \\
\vec{m} \\
\end{array}$ & $\begin{array}{l}10 \\
\infty \\
\infty \\
0 \\
0 \\
0 \\
\infty \\
0 \\
\end{array}$ & $\begin{array}{l}10 \\
0 \\
0 \\
+ \\
0 \\
0 \\
0 \\
+ \\
+\end{array}$ & 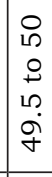 & 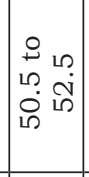 \\
\hline نे & $\vec{u}$ & $\vec{u}$ & $\vec{u}$ & ש & $\tilde{z}^{2}$ & U3 & 己 & 10 & U & U \\
\hline 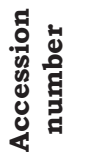 & 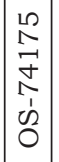 & $\begin{array}{l}0 \\
2 \\
2 \\
1 \\
1 \\
0 \\
2 \\
0\end{array}$ & 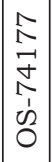 & \begin{tabular}{l}
0 \\
2 \\
\multirow{5}{\alpha}{} \\
2 \\
1 \\
0 \\
0
\end{tabular} & $\begin{array}{l}\stackrel{0}{1} \\
0 \\
0 \\
0 \\
1 \\
1 \\
02 \\
0\end{array}$ & $\begin{array}{l}\sigma \\
\overrightarrow{0} \\
0 \\
1 \\
\dot{1} \\
0\end{array}$ & $\begin{array}{l}\vec{a} \\
1 \\
0 \\
0 \\
1 \\
\dot{1} \\
0\end{array}$ & \begin{tabular}{l}
0 \\
$\infty$ \\
$y$ \\
\multirow{1}{1}{} \\
$\dot{j}$ \\
$\hat{2}$ \\
0
\end{tabular} & 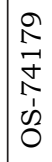 & $\begin{array}{l}\vec{N} \\
\text { Oे } \\
0 \\
\infty \\
\dot{1} \\
0\end{array}$ \\
\hline 总 & -1 & o & $\infty$ & + & in & 0 & $\wedge$ & $\infty$ & $a$ & $\stackrel{-}{-1}$ \\
\hline
\end{tabular}




\begin{tabular}{|c|c|c|c|c|c|c|c|c|c|c|c|c|c|c|c|c|c|c|}
\hline $\begin{array}{l}0 \\
\infty \\
+1 \\
\llcorner \\
\sigma \\
\sigma \\
\wedge\end{array}$ & & - & 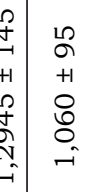 & & $\begin{array}{l}2 \\
0 \\
+1 \\
+1 \\
0 \\
\overrightarrow{6}\end{array}$ & & $\begin{array}{l}L \\
L \\
L \\
+1 \\
L \\
+ \\
O \\
- \\
-\end{array}$ & 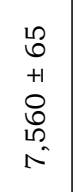 & & 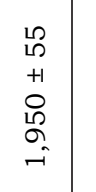 & $\begin{array}{l}8 \\
+1 \\
+ \\
\infty \\
\infty \\
1\end{array}$ & & $\begin{array}{l}8 \\
0 \\
+1 \\
10 \\
0 \\
0 \\
\infty\end{array}$ & & $\begin{array}{l}0 \\
0 \\
+1 \\
0 \\
+ \\
10 \\
-1\end{array}$ & & & \\
\hline 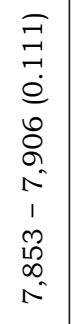 & 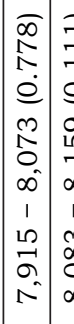 & 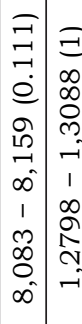 & 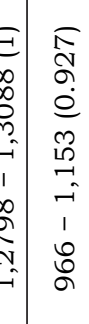 & 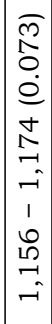 & 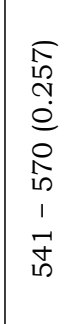 & 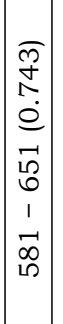 & 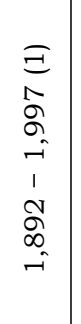 & 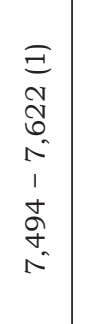 & & 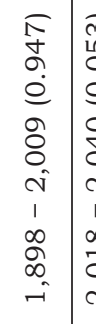 & 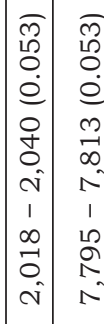 & 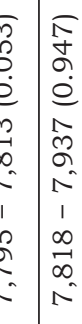 & 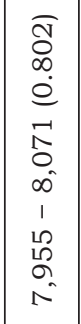 & $\begin{array}{c}0 \\
0 \\
2 \\
-1 \\
0 \\
\infty \\
1 \\
0 \\
\infty \\
1 \\
1 \\
0 \\
\infty \\
0 \\
\infty \\
\infty\end{array}$ & 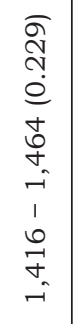 & 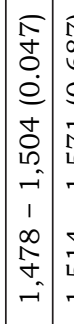 & 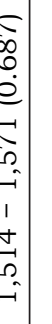 & 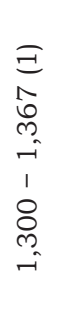 \\
\hline 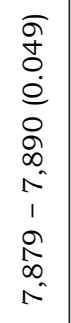 & $\begin{array}{c}\overrightarrow{1} \\
\hat{2} \\
0 \\
0 \\
0 \\
0 \\
0 \\
\infty \\
1 \\
0 \\
0 \\
0 \\
\hat{N}\end{array}$ & $\begin{array}{l}\Xi \\
\infty \\
\infty \\
0 \\
0 \\
0 \\
-1 \\
1 \\
\infty \\
\infty \\
\infty \\
N \\
-1\end{array}$ & 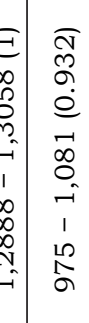 & 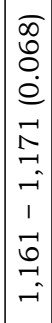 & 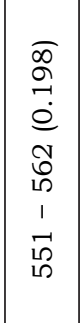 & $\left|\begin{array}{c}\widehat{y} \\
0 \\
0 \\
0 \\
\hat{0} \\
\hat{0} \\
1 \\
0 \\
\delta \\
\hat{n}\end{array}\right|$ & $\begin{array}{l}\Xi \\
\infty \\
\infty \\
0 \\
-1 \\
1 \\
0 \\
\sigma \\
\sigma \\
- \\
-\end{array}$ & 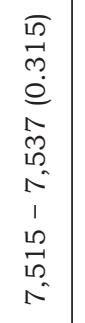 & 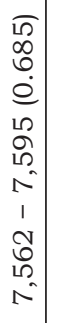 & 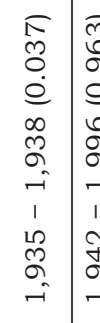 & 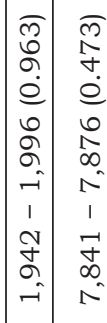 & 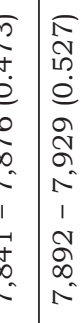 & 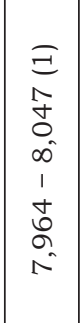 & & 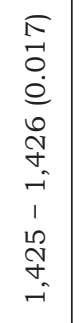 & 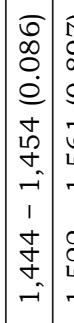 & 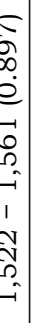 & 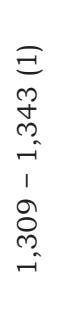 \\
\hline 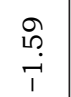 & & $\begin{array}{l}\delta \\
0 \\
\infty \\
1\end{array}$ & 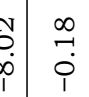 & & $\stackrel{\leftrightarrow}{i}$ & & $\begin{array}{l}\text { ते } \\
\text { Oे }\end{array}$ & $\begin{array}{l}\mathfrak{N} \\
\hat{i} \\
\dot{1}\end{array}$ & & $\begin{array}{l}\infty \\
\infty \\
\infty \\
0\end{array}$ & $\stackrel{\text { I }}{\stackrel{0}{0}}$ & & $\begin{array}{l}m \\
0 \\
\dot{0} \\
1\end{array}$ & & $\vec{i}$ & & & 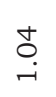 \\
\hline 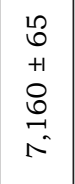 & & $\begin{array}{l}0 \\
0 \\
+1 \\
8 \\
0 \\
= \\
=\end{array}$ & 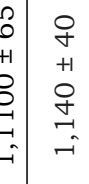 & & 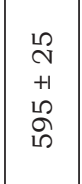 & & $\begin{array}{l}\stackrel{\sim}{n} \\
\stackrel{1}{+} \\
+1 \\
\& \\
\& \\
i\end{array}$ & $\begin{array}{l}10 \\
0 \\
+1 \\
8 \\
8 \\
0 \\
0\end{array}$ & & $\begin{array}{l}\stackrel{L}{n} \\
+1 \\
+\infty \\
o \\
o \\
i \\
i\end{array}$ & $\begin{array}{l}\circ \\
\infty \\
+1 \\
0 \\
\varnothing \\
0 \\
N\end{array}$ & 要 & $\begin{array}{l}o \\
+ \\
+1 \\
o \\
\vec{N} \\
N\end{array}$ & & $\begin{array}{l}\stackrel{1}{N} \\
+1 \\
+1 \\
0 \\
0 \\
0 \\
-\end{array}$ & & & 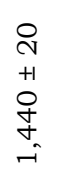 \\
\hline $\begin{array}{l}\infty \\
\tilde{0} \\
0 \\
\dot{0} \\
+1 \\
0 \\
\overrightarrow{+} \\
0\end{array}$ & & 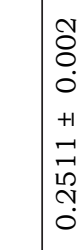 & 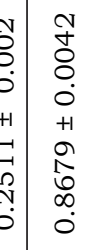 & & 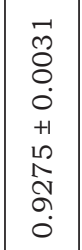 & & 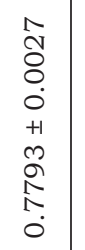 & 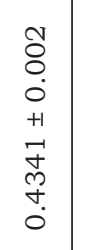 & & 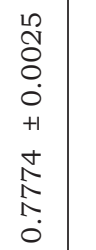 & 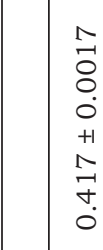 & & $\begin{array}{l}0 \\
\overrightarrow{8} \\
0 \\
0 \\
+1 \\
0 \\
0 \\
0 \\
\dot{0} \\
0\end{array}$ & & $\begin{array}{l}\hat{\widehat{N}} \\
0 \\
0 \\
0 \\
++1 \\
\hat{0} \\
0 \\
0 \\
0\end{array}$ & & & 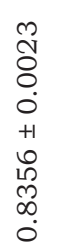 \\
\hline 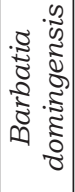 & & 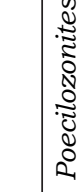 & 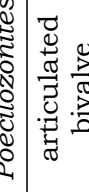 & & 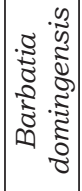 & & 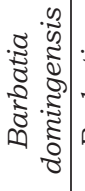 & 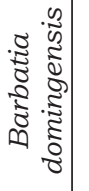 & & 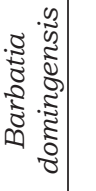 & 通 & 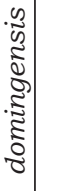 & 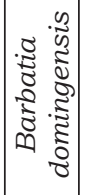 & & 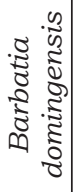 & & & 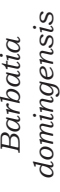 \\
\hline 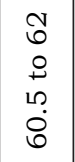 & & $\begin{array}{l}10 \\
10 \\
0 \\
0 \\
0 \\
10 \\
0\end{array}$ & 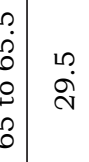 & & $\begin{array}{l}\mathcal{N} \\
\stackrel{8}{\circ} \\
\exists\end{array}$ & & 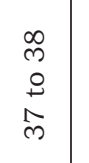 & $\begin{array}{l}8 \\
8 \\
0 \\
0 \\
0\end{array}$ & & $\begin{array}{l}\text { مִ } \\
\text { m } \\
\stackrel{0}{0} \\
\hat{m}\end{array}$ & \begin{tabular}{l} 
I \\
\& \\
\multirow{+}{*}{}
\end{tabular} & $\begin{array}{l}\text { y } \\
\text { y } \\
\text { o }\end{array}$ & 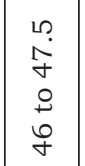 & & $\begin{array}{l}\text { Oे } \\
\stackrel{0}{1} \\
\text { ते }\end{array}$ & & & $a$ \\
\hline 0 & & $\stackrel{10}{0}$ & $\begin{array}{l}0 \\
\mathcal{U}\end{array}$ & & i & & ర & i & & $\vec{u}$ & $\vec{v}$ & & $\vec{u}$ & & $\stackrel{\mathcal{N}}{\vec{u}}$ & & & $\stackrel{m}{u}$ \\
\hline $\begin{array}{l}\infty \\
\vec{N} \\
\widehat{N} \\
1 \\
\tilde{y} \\
0\end{array}$ & & 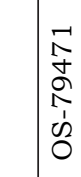 & 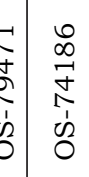 & & $\begin{array}{l}\text { Oे } \\
0 \\
0 \\
\infty \\
0 \\
1^{2} \\
0\end{array}$ & & $\begin{array}{l}\checkmark \\
0 \\
m \\
-1 \\
\infty \\
1 \\
0 \\
0\end{array}$ & $\begin{array}{l}0 \\
0 \\
0 \\
0 \\
1 \\
1 \\
0\end{array}$ & & $\begin{array}{l}00 \\
0 \\
0 \\
\infty \\
0 \\
1 \\
0 \\
0\end{array}$ & $\begin{array}{l}0 \\
0 \\
0 \\
-1 \\
0 \\
1 \\
1 \\
0\end{array}$ & $\begin{array}{l}0 \\
0 \\
0 \\
0 \\
0 \\
b\end{array}$ & $\begin{array}{l}0 \\
0 \\
0 \\
0 \\
0 \\
1 \\
0 \\
0\end{array}$ & & $\begin{array}{l}\hat{0} \\
0 \\
0 \\
0 \\
1 \\
0 \\
0\end{array}$ & & & 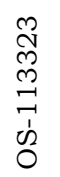 \\
\hline$\exists$ & & $\underset{7}{7}$ & 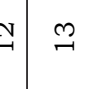 & & $\stackrel{ \pm}{*}$ & & $\stackrel{2}{\rightarrow}$ & $\stackrel{0}{\sim}$ & & $\stackrel{\curvearrowright}{\neg}$ & $\stackrel{\infty}{\sim}$ & to & $\stackrel{2}{7}$ & & กิ & & & $\sim$ \\
\hline
\end{tabular}


geophysical, and paleoenvironmental analysis in Harrington Sound, and documented how Harrington Sound was originally an isolated and stratified marine pond in the early to middle Holocene. Appearance of Oculina coral in the uppermost stratigraphy from Harrington Sound is thought to represent the onset of modern coastal circulation between Harrington Sound and North Shore Lagoon through Flatts Inlet. The onset of carbonate deposition in Green Bay Cave was interpreted by van Hengstum et al. (2011) as likely related to the onset of oceanic circulation between Harrington Sound and North Shore Lagoon through Flatts Inlet.

The eight sediment cores for the present study only sampled a carbonate facies similar to that documented by van Hengstum et al. (2011), and indicates that all the recovered stratigraphy for the present study is likely less than 2,000 years old (Fig. 3). Indeed, the radiocarbon date at the base of core 13 in the carbonate mud is $\sim 1,335 \mathrm{Cal}$ yrs BP, which is consistent with our previous results (Table 1). In general, the carbonate mud had a mean grain size of $<30 \mu \mathrm{m}$ (medium silt) and contained approximately $12 \%$ bulk organic matter, with some spatial textural variability occurring between the core sites. The greatest grai nsize increase and bulk organic matter changes were observed at depth in cores located most proximal to the Letter Box (core 13 and 17). Core 15, positioned more proximal to the submarine entrance into Harrington Sound, had a minimal shift in mean grain size, from $<30 \mu \mathrm{m}$ to approximately $80 \mu \mathrm{m}$ (very fine sand) and with no significant change in organic matter (Fig. 4).

Cores 13 and 17 contained changes in sediment texture that were distinctly different from the typical late Holocene carbonate mud facies. In core 13, a prominent carbonate sand layer from 17 to $22 \mathrm{~cm}$ $(\sim 5 \mathrm{~cm}$ thick) with decreased bulk organic matter, abruptly transitioned into a $1 \mathrm{~cm}$ thick layer of coarse-grained organic matter particles (Fig. 5). The

A
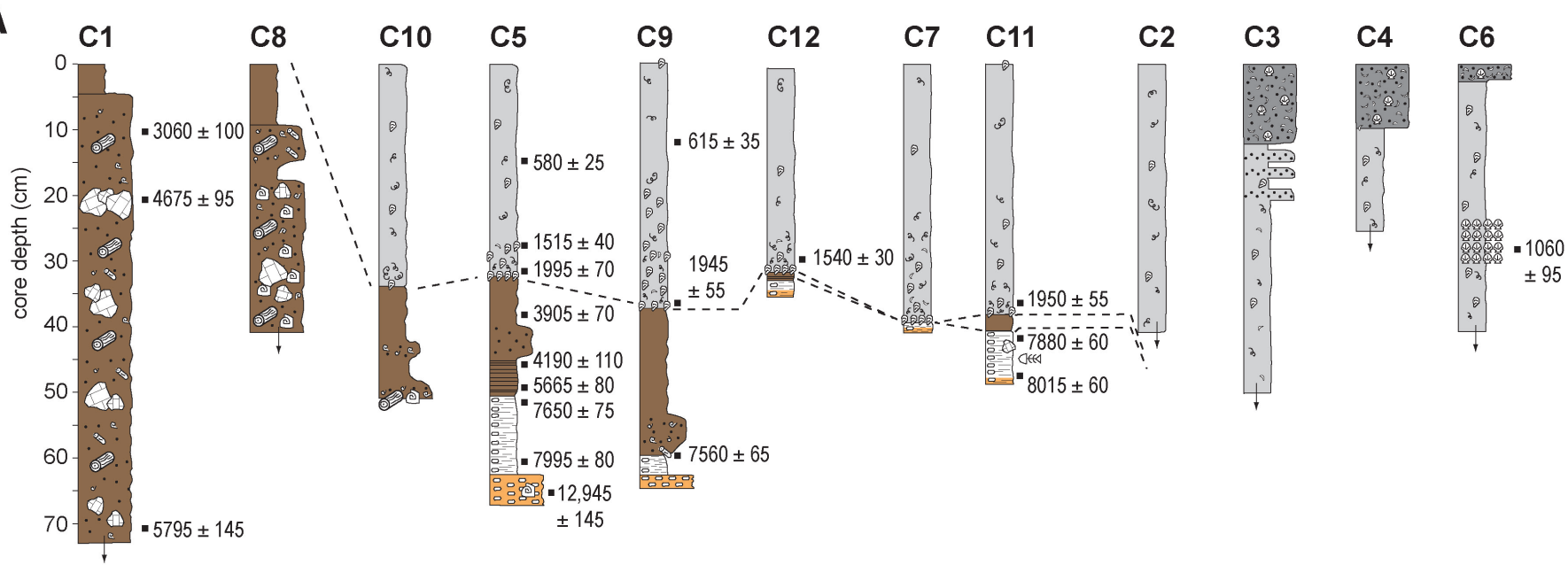

B
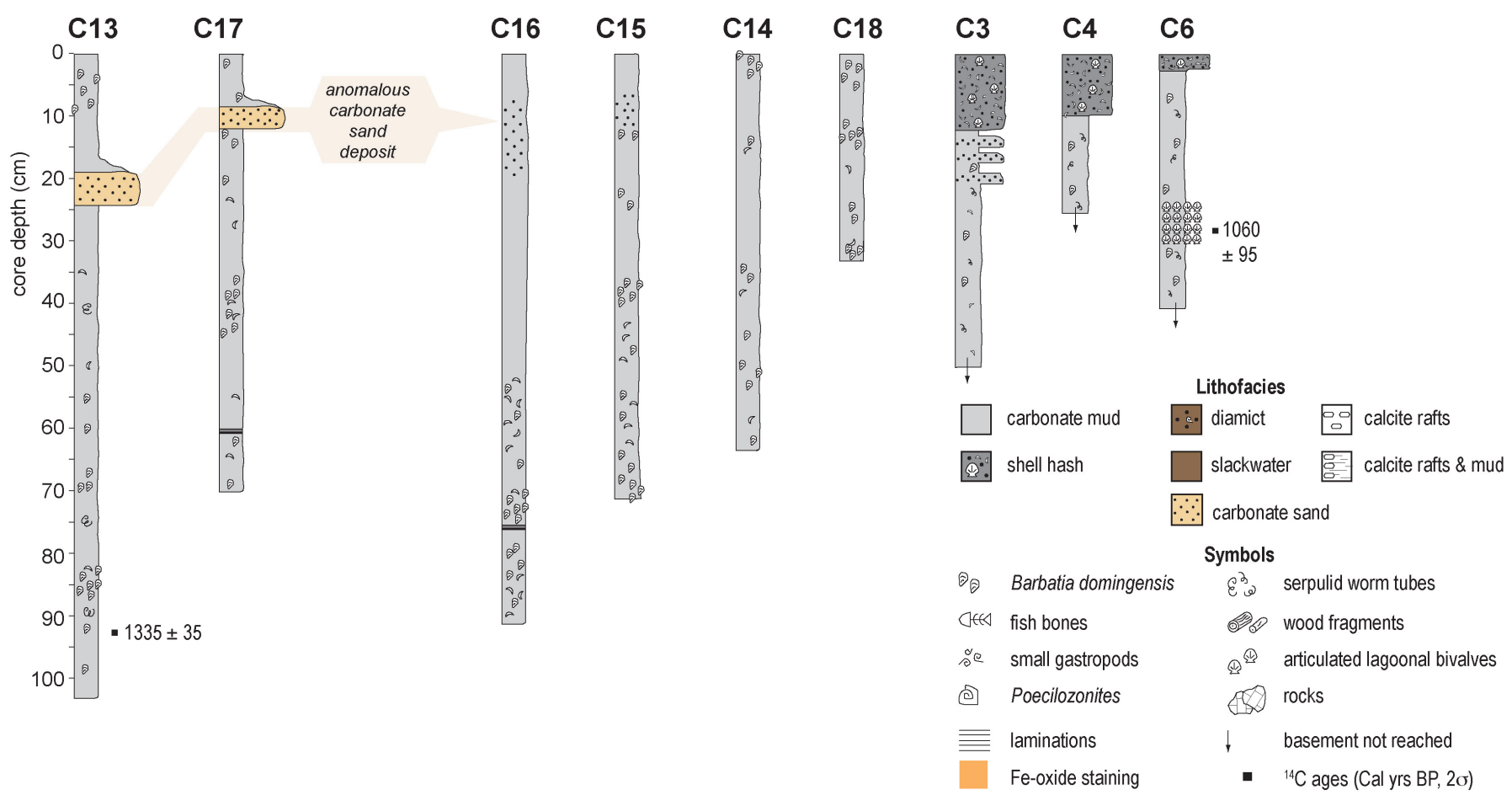

Fig. 3. Stratigraphic columns for all sediment cores collected from Green Bay Cave, including cores 1-12 (van Hengstum et al., 2011) and cores 13-18 (this study). Note that all conventional radiocarbon ages have been calibrated with IntCal13 (Reimer et al., 2013). 


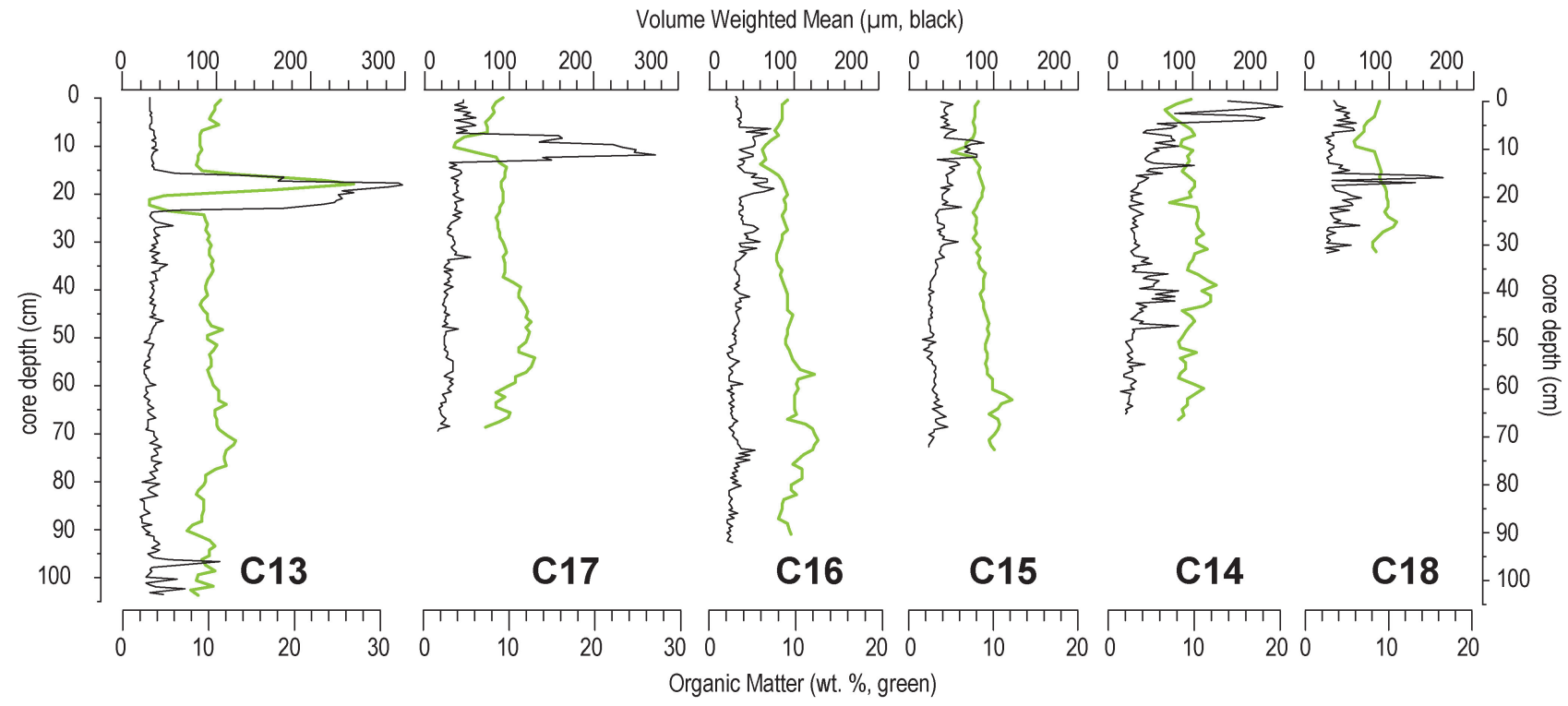

Fig. 4. Detailed X-radiograph and photograph of the anomalous carbonate sand unit in core 13 (top) and 17 (bottom).

mean grain size increased from $\sim 30 \mu \mathrm{m}$ (medium silt) below $22 \mathrm{~cm}$, to $\sim 240 \mu \mathrm{m}$ (medium sand) from 22 to $18 \mathrm{~cm}$, and then decreased to $30 \mu \mathrm{m}$ again above $18 \mathrm{~cm}$. The bulk organic matter content also abruptly shifted from $\sim 12$ to $\sim 4 \%$ in the carbonate sand layer at 18 to $22 \mathrm{~cm}$, subsequently increasing to $\sim 30 \%$ from 18 to $15 \mathrm{~cm}$, and then reverting back to $\sim 12 \%$ from $15 \mathrm{~cm}$ to the coretop. This organic matter layer contained coarser-grained organic matter particles derived from plant material. Similarly, core 17 also had a carbonate sand layer from 12 to $9 \mathrm{~cm}$. Mean grain size in core 17 increased from $30 \mu \mathrm{m}$ below $12 \mathrm{~cm}$, to $245 \mu \mathrm{m}$ between 9 to $12 \mathrm{~cm}$, and decreased to $<30 \mu \mathrm{m}$ from $9 \mathrm{~cm}$ to the coretop. In addition, bulk organic matter content decreased from 12 to $5 \%$ between 12 to $9 \mathrm{~cm}$, before immediately returning to $12 \%$, from $9 \mathrm{~cm}$ to the coretop. This carbonate sand layer is anomalous with respect to the entire Holocene succession known from Green Bay Cave (van Hengstum et al., 2011) or late Holocene successions in nearby Walsingham Cavern (van Hengstum et al., 2015b).

\section{Benthic foraminifera}

Based on a detailed diversity analysis of foraminifera in core 13, four biofacies could be identified in the dendrogram produced by stratigraphicallyconstrained Q-mode cluster analysis at a Euclidean distance of 3.2 (Fig. 6). These biofacies were characterized by similar dominant foraminifera adapted to specific environmental conditions, which were interpreted to represent intervals of time with similar hydrological and environmental conditions in the cave (Fig. 7). In stratigraphic order (oldest to youngest) the biofacies were: (i) Low Oxic Submarine Assemblage, (ii) Circulated Submarine Assemblage, (iii) the Impact Assemblage, and (iv) the Recovery/ Circulated Submarine Assemblage, so named for their consistency with previous results of van Hengstum \& Scott (2012).

The Low Oxic Submarine Assemblage, located from 100 to $80 \mathrm{~cm}$ in core $13(n=5)$, had the second lowest diversity $(H=2.733)$ and the lowest species richness $(\mathrm{R}=35)$. The age at $93 \mathrm{~cm}$ in core 13 was $1,335 \mathrm{Cal} \mathrm{yrs}$

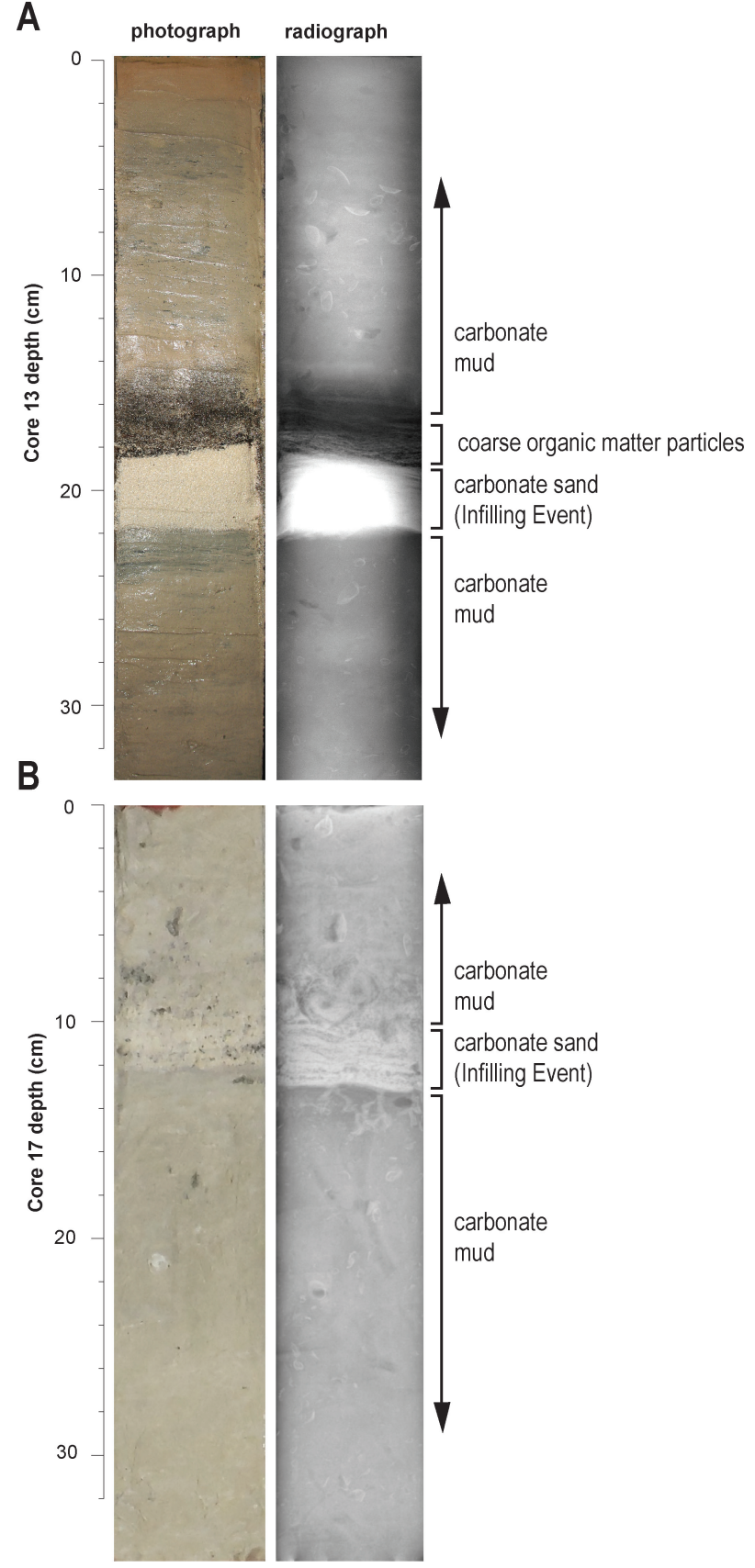

Fig. 5. Downcore variability in bulk organic matter and mean grain size from cores in the transect from $A$ to $B$ in Fig. 1B. 
$\mathrm{BP}$, which was just centuries after circulation likely initiated between Green Bay Cave and Harrington Sound (van Hengstum et al., 2011; van Hengstum $\&$ Scott, 2012). This assemblage is dominated by infaunal biserial taxa like Bolivina variabilis (mean 19\%), epifaunal rotaliids Rosalina spp. (mean 12.4\%), and Suratkina australiensis (mean 9.2\%, Table 2).

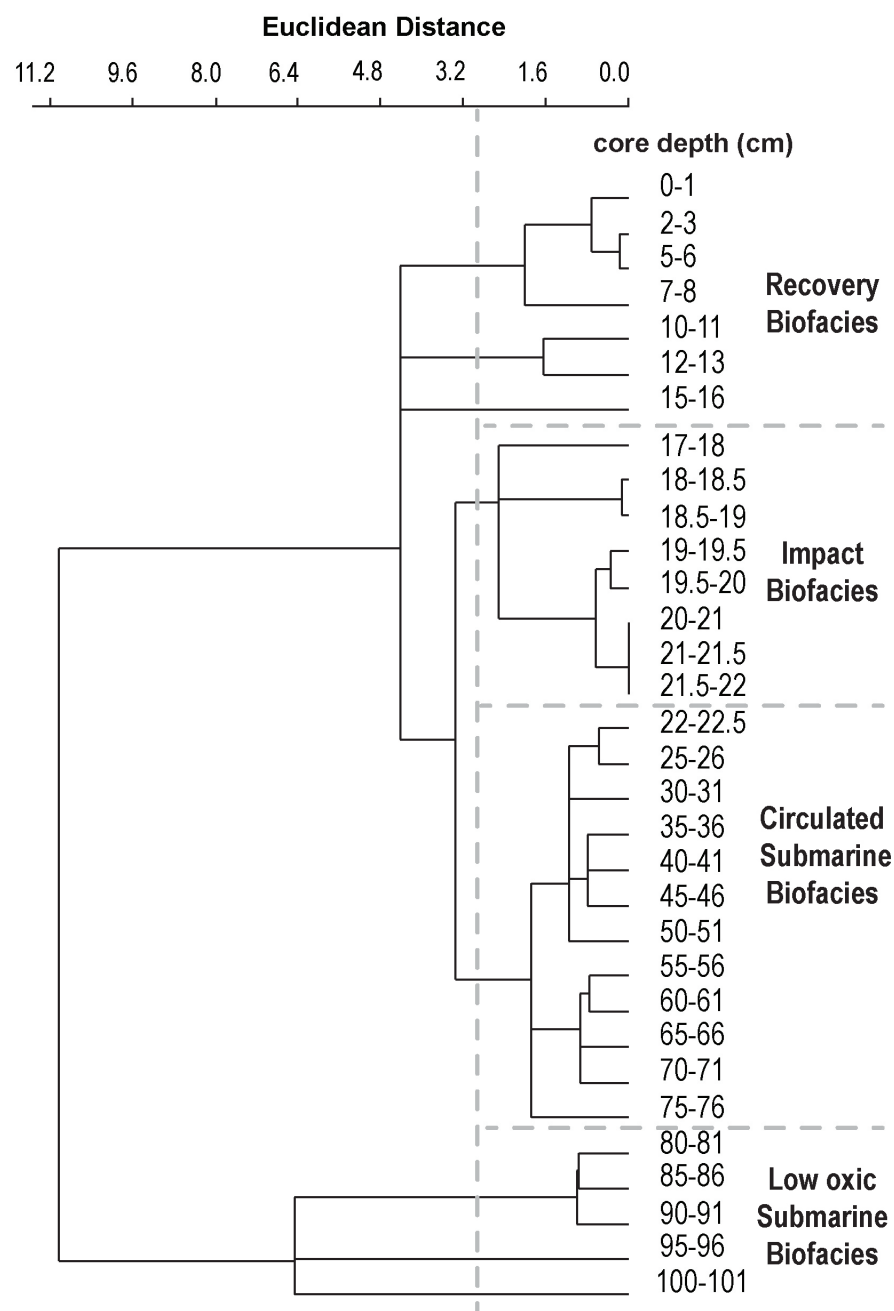

Fig. 6. Dendrogram produced by the Q-mode cluster analysis, and subsequent biofacies identification.
The Circulated Submarine Assemblage, located from 80 to $22 \mathrm{~cm}$ in core 13 ( $n=12$ total samples), had a foraminiferal population that was notably diversified from lower positions in the core $(H=3.22, \mathrm{R}=49)$. This assemblage was dominated by the miliolid genera Quinqueloculina (mean 9.9\%), Miliolinella spp. (mean 9.6\%), and Spirophthalmidium emaciatum (mean 6.8\%), with decreased rotaliid abundance (e.g., Bolivina, Rosalina, and Suratkina).

The Impact Assemblage, present in the anomalous carbonate sand layer between 22 and $17 \mathrm{~cm}$ in core $13(n=8)$, had the lowest diversity among all assemblages $(H=2.5, \mathrm{R}=50)$. It was dominated by Quinqueloculina spp. (mean 39.7\%) and Cibicides sp. (mean $7.9 \%$ ), and the symbiont-bearing foraminifer Peneroplis pertusus was rare, but observed (Fig. 8). Taxa that were abundant in the carbonate mud facies abruptly decreased, such as Sigmoilina tenuis, Patellina corrugata, Spirophthalmidium emaciatum (mean 1.7\%), and Rosalina spp. (mean 3.34\%).

The Recovery/Circulated Submarine Assemblage, found between $17 \mathrm{~cm}$ and the coretop ( $n=7$ total samples), was characterized by increased diversity and species richness relative to the lower Impact Assemblage $(H=3.22 ; \mathrm{R}=53)$. This assemblage was dominated by Quinqueloculina spp. (mean 13.4\%), Rosalina spp. (mean 8.9\%), and Spirophthalmidium emaciatum (mean 9.4\%), with a complete loss of Cibicides sp. and Peneroplis spp. Additionally, many benthic foraminiferal taxa, previously observed in the Circulated Submarine Assemblage from 80 to $22 \mathrm{~cm}$, recovered to relative abundance levels that existed prior to the Impact Assemblage (e.g., Cyclogyra involvens: mean 2.9\%, Sigmoilina tenuis: mean $2.9 \%$ ). The diversity and species composition of samples comprising the Recovery/Circulated Submarine Cave Assemblage in core 13 were consistent with benthic foraminifera currently present at the sediment-water interface in areas of Green Bay Cave that are wellcirculated with marine water from outside the cave setting (van Hengstum \& Scott, 2011).

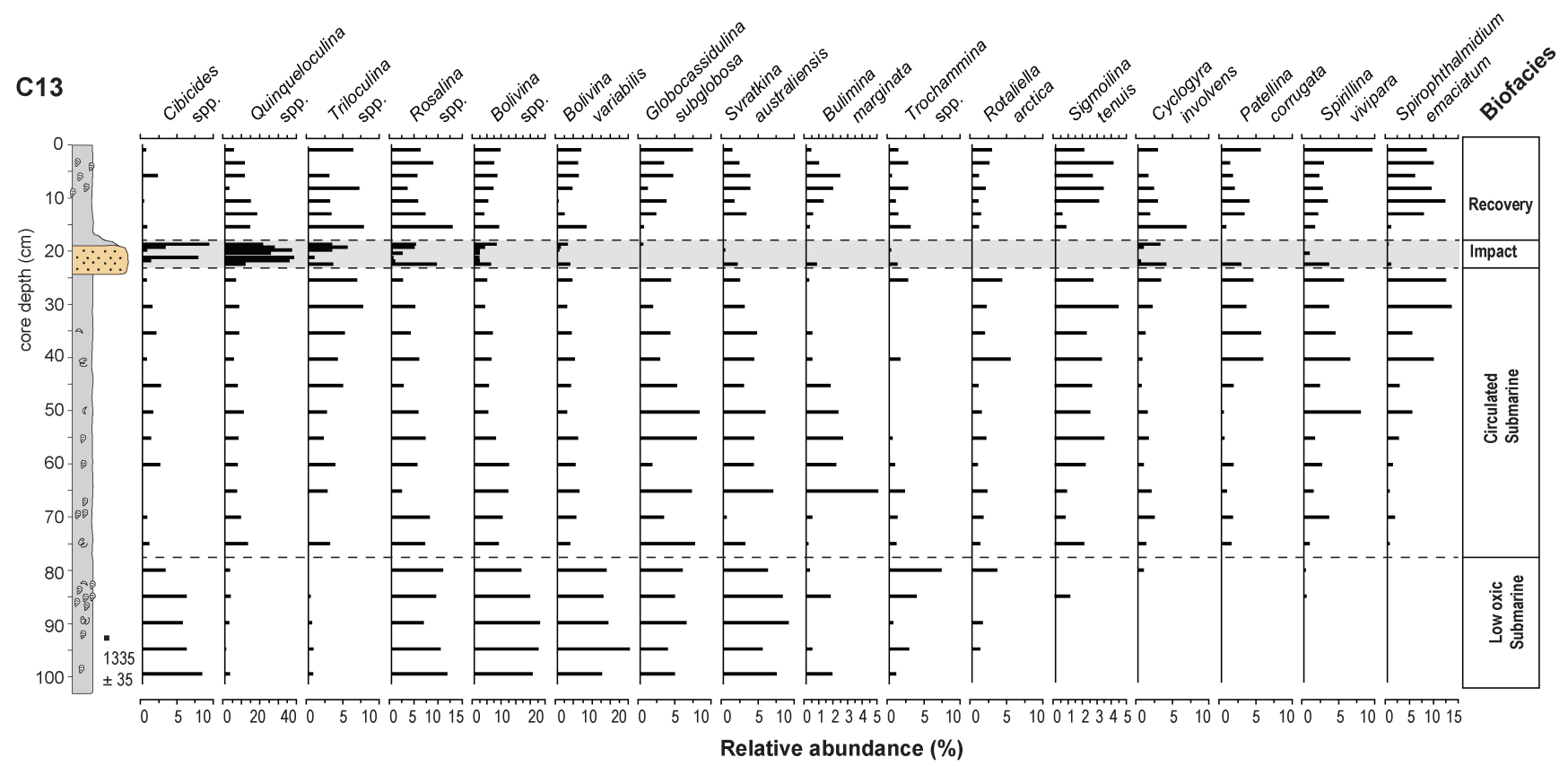

Fig. 7. Detailed lithology, radiocarbon date, relative abundance of dominant benthic foraminifera, and biofacies from core 13. 
Table 2. Arithmetic mean of the relative abundance of dominant taxa and textural characteristics for each biofacies. Species with a mean of $<1 \%$ relative abundance in the biofacies were marked with a dash so dominant species could be emphasized.

\begin{tabular}{|c|c|c|c|c|}
\hline Biofacies & Low Oxic & $\begin{array}{l}\text { Circulated } \\
\text { Submarine }\end{array}$ & Impact Event & $\begin{array}{c}\text { Recovery/Circulated } \\
\text { Submarine }\end{array}$ \\
\hline Approximate Timeframe & $\sim 1300 \mathrm{Cal}$ Yrs BP & Last millennium & Mid-20 ${ }^{\text {th }}$ Century & Mid-20 ${ }^{\text {th }}$ Century until present \\
\hline \multicolumn{5}{|l|}{ Sediment Properties } \\
\hline Mean grain size (um) & 29.895 & 30.577 & 232.233 & 32.404 \\
\hline Organic matter (\%) & 8.629 & 9.847 & 9.484 & 10.052 \\
\hline \multicolumn{5}{|l|}{ Foraminifera } \\
\hline Total individuals $\left(\mathrm{cm}^{3}\right)$ & 1,428 & 4,335 & 1,664 & 2,438 \\
\hline Shannon-Wiener Diversity Index $(H)$ & 2.733 & 3.224 & 2.507 & 3.224 \\
\hline Species Richness ( R ) & 35 & 49 & 50 & 53 \\
\hline \multicolumn{5}{|l|}{ Relative Abundance } \\
\hline Bolivina variabilis & 19.0 & 5.2 & 1.5 & 6.2 \\
\hline Bolivina spp. & 7.1 & 3.2 & 4.9 & 2.5 \\
\hline Bulimina marginata & - & 1.5 & - & 1.3 \\
\hline Cibicides spp. & 7.5 & 1.5 & 7.9 & 1.2 \\
\hline Cyclogyra involvens & - & 2.2 & 1.5 & 2.9 \\
\hline Elphidium spp. & - & - & 2.1 & - \\
\hline Globocassidulina subglobosa & 6.6 & 6.0 & - & 4.4 \\
\hline Melonis barleeanum & 6.1 & 5.2 & - & 4.6 \\
\hline Miliolinella spp. & - & 9.6 & 1.2 & 5.8 \\
\hline Nonion spp. & 8.5 & 3.3 & 5.3 & 1.5 \\
\hline Patellina corrugata & - & 3.3 & - & 3.2 \\
\hline Peneroplis spp. & - & - & 6.7 & - \\
\hline Quinqueloculina spp. & 2.9 & 9.9 & 39.7 & 13.4 \\
\hline Rotaliella arctica & 1.7 & 2.8 & 2.2 & 2.1 \\
\hline Rosalina spp. & 12.4 & 6.3 & 5.3 & 8.9 \\
\hline Spirillina vivipara & - & 4.9 & - & 4.6 \\
\hline Sigmoilina tenuis & - & 2.9 & - & 2.9 \\
\hline Spirophthalmidium emaciatum & - & 6.8 & 2.2 & 9.4 \\
\hline Suratkina australiensis & 9.2 & 4.4 & - & 2.7 \\
\hline Triloculina spp. & - & 5.0 & 4.6 & 5.0 \\
\hline Trochammina spp. & 3.9 & 1.3 & 1.6 & 2.3 \\
\hline Sum & 84.9 & 85.4 & 86.7 & 84.9 \\
\hline
\end{tabular}

\section{DISCUSSION}

\section{Pre-impact environmental conditions in Green Bay Cave}

Benthic foraminifera that were ecologically successful (i.e., high relative abundance) $\sim 1,300$ years ago at the base of the Letter Box were tolerant of lower dissolved oxygen levels. Vollbrecht (1996) proposed that inundation of the channel between Harrington Sound and the North Shore Lagoon (Flatt's Inlet) by Holocene sea-level rise likely increased circulation between the two water bodies. van Hengstum et al. (2011) suggested that this event also increased the circulation of seawater between Harrington Sound and Green Bay Cave, and was linked to the onset of carbonate mud deposition in Green Bay Cave. The Low Oxic Assemblage at the base of core 13 was dominated by members of the genus Bolivina (mean $19 \%$ ), which have a biserial chamber arrangement. In general, benthic foraminifera with a biserial chamber arrangement are adapted to an infaunal life mode, where dissolved oxygen concentrations below the sediment-water interface are lower relative to typical benthic seawater (Jorissen, 1999). Foraminifera with a biserial chamber arrangement are also dominant in benthic habitats with a high organic matter flux because microbial degradation of organic matter can decrease dissolved oxygen concentrations at the sedimentwater interface. As such, assemblages dominated by bolivinids are typically found in benthic environments with lower dissolved oxygen concentrations (Kaiho, 1994; Bernhard \& Gupta, 1999; Jorissen, 1999). In addition, the test of subfossil Suratkina australiensis, which was also common in the Low Oxic Assemblage, was characterized by an abundance of large pores on the test surface. Increased number and size of pores on the test of benthic foraminifera is also considered an adaptation to low dissolved oxygen concentrations, thought to enhance dissolved gas exchange between the organism and seawater (Leutenegger \& Hansen, 1979; Petersen et al., 2016). These results suggest that the benthic foraminifera 


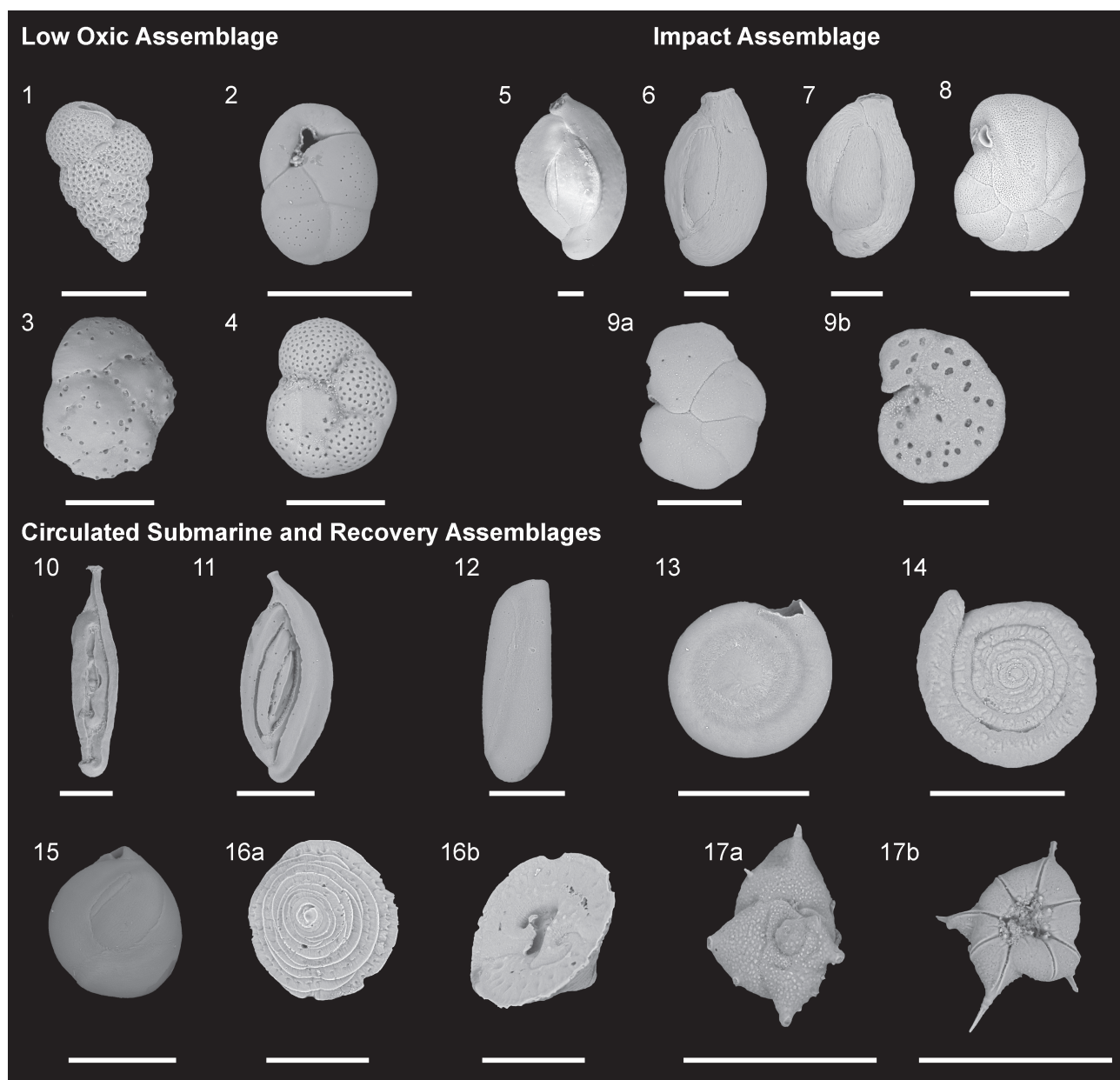

Fig. 8. Representative benthic foraminifera recovered from Green Bay Cave, Core 13. Low oxic biofacies (1 to 4): 1) Bolivina variabilis (Williamson, 1858); 2) Globocassidulina subglobosa (Brady, 1881); 3) Svratkina australiensis; 4) Rosalina globularis; Impact biofacies (5 to 10): 5-7) Quinqueloculina spp.; 8) Peneroplis pertusus; 9) Cibicides lobatulus (a: dorsal; b: ventral); Circulated and Recovery biofacies (11 to 17): 10) Spirophthalmidium emaciatum (Haynes, 1973); 11) Sigmoilina tenuis (Czjzek, 1848); 12) Triloculina oblonga (Montagu, 1803); 13) Cyclogyra involvens; 14) Spirillina vivipara Ehrenberg (Ehrehberg, 1843); 15) Miliolinella circularis; 16) Patellina corrugata (a: dorsal; b: ventral); 17) Rotaliella arctica (a: dorsal; b: ventral) (Scott \& Vilks, 1991). All scale bars represent $100 \mathrm{~mm}$.

colonizing the passage below the Letter Box in Green Bay Cave $\sim 1,330$ years ago were adapted to low-oxic marine conditions (Fig. 9A). Elsewhere in Green Bay Cave (i.e., Trunk Passage), van Hengstum \& Scott (2012) found similar contemporaneous assemblages of benthic foraminifera, which indicate that the saline groundwater flooding the cave had dissolved oxygen levels $\sim 1,300$ years ago that were in the low oxic range (1.5-3 ml/1).

The benthic foraminifera transition upcore into the Circulated Submarine Assemblage, which was dominated by taxa comparable to modern fauna in Green Bay Cave that are relatively more impacted by tidal exchange of seawater through the submarine cave entrance (van Hengstum \& Scott, 2011). This change in foraminifera upcore indicate a change in circulation of saline groundwater through the cave, which would have altered the relative flux of either (i) dissolved or particulate matter imported into the cave from adjacent coastal areas, or (ii) change the exposure of the benthic environment to water upwelling through the carbonate bedrock (Fig. 9B). Elsewhere, van Hengstum et al. (2015b) used tidal and seasonal temperature changes of the saline groundwater in Walsingham Cavern as a proxy to document how seawater circulates through the subsurface and into the cave from both the Sargasso Sea and adjacent coastal lagoons in response to tidal forcing. We speculate that similar physical circulation mechanisms are also operant in Green Bay Cave, but remain uncertain as to which process may have more significantly impacted the benthic foraminifera at the onset of the Circulation Submaine Assemblage.

Dominant taxa of the Circulated Submarine Assemblage were all members of the miliolid group (e.g., Spirophthalmidium, Miliolinella, and Quinqueloculina), which are dominant in well-oxygenated, tropical and subtropical carbonate lagoons. The exception was Spiropthalmidium emaciatum, which is dominant throughout Bermuda's flooded marine caves (i.e., stygophile). The Circulated Submarine Assemblage characterized the pre-anthropogenic, natural benthic foraminiferal community in Green Bay Cave as circulation between seawater from Harrington Sound and the saline groundwater stabilized over the last millennium.

\section{Stratigraphic evidence for the infilling of Eve's Pond}

The abrupt change to carbonate sand in core 13 and 17 with a texture similar to carbonate sand in modern lagoons, a decrease in stygophilic benthic 
foraminifera (e.g., Spiropthalmidium emaciatum, Sigmoilina tenuius), and an increase in lagoon foraminifera indicate that the anomalous carbonate sand layer was most likely related to the infilling of Eve's Pond in the mid-20 $0^{\text {th }}$ century (Fig. 9C). This infilling event introduced foreign sediment into Green Bay Cave, forming a spatially discontinuous deposit. Currently, carbonate sediment with a mean grain size of $30 \mu \mathrm{m}$ (silt) dominated all cores sampled along the impacted cave passage and was similar in grainsize

A Low Oxic Submarine Cave Environment: 1335 Cal yrs BP

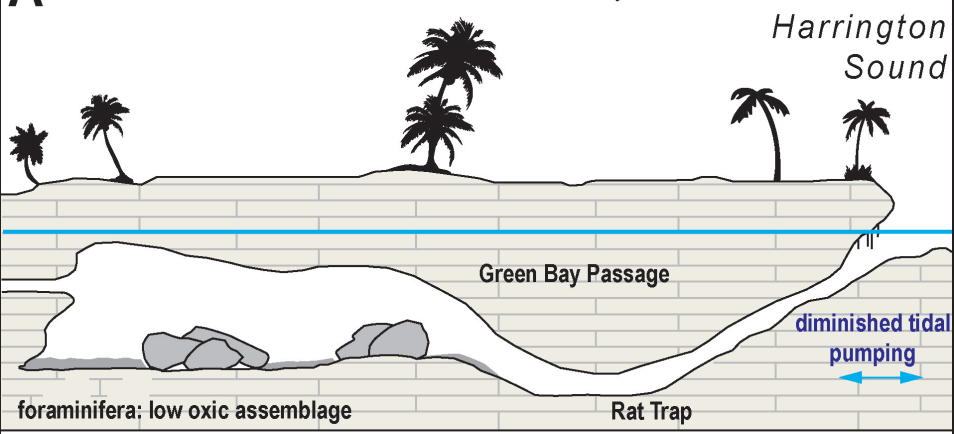

B Circulated Submarine Cave Environment: Last millenium

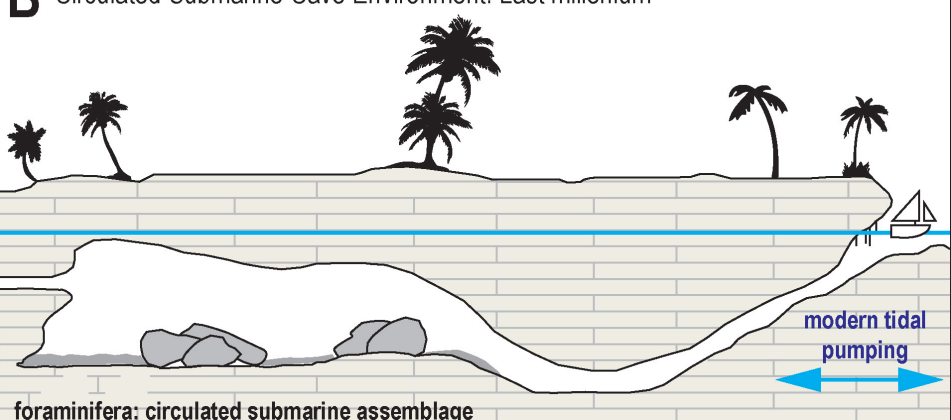

foraminifera: circulated submarine assemblage

C Impact Event (middle 20 ${ }^{\text {th }}$ century)

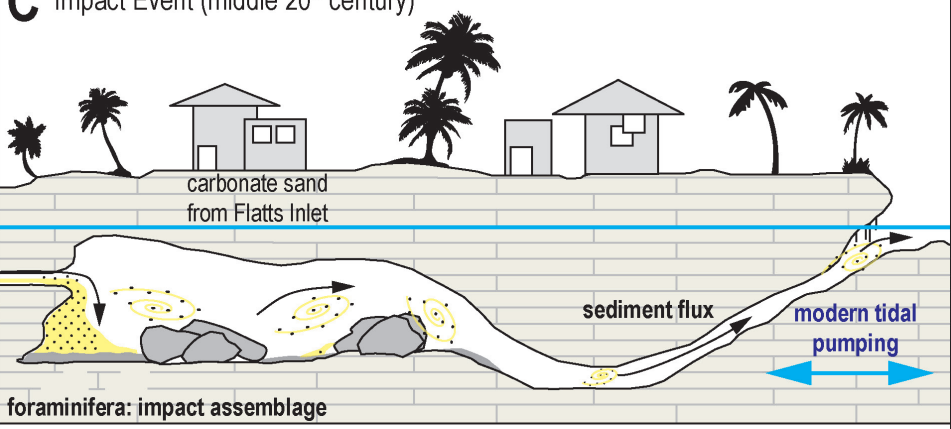

foraminifera: impact assemblage

Decovery/Circulated Submarine Cave Environment

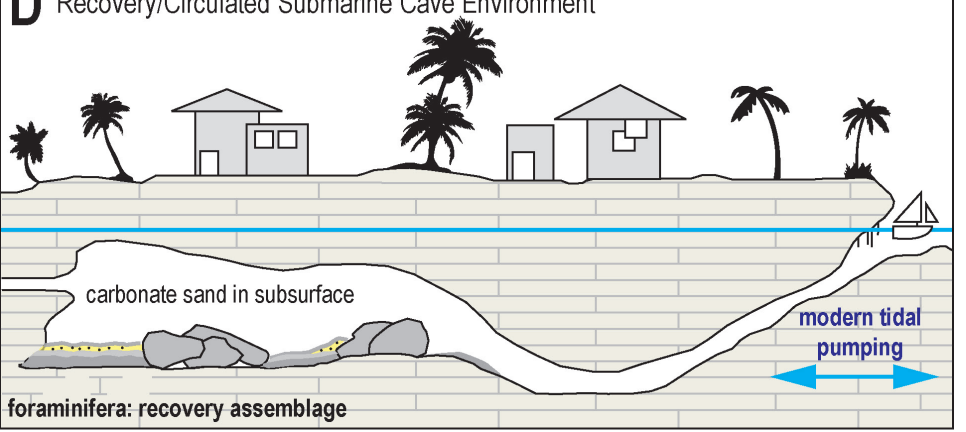

Fig. 9. A conceptual model documenting long-term environmental change in the cave passage from Green Bay to the Letter Box area. A) The uninhabited Bermuda landscape with more limited circulation of water between Harrington Sound and Green Bay Cave; B) evidence for modern circulation patterns in the Green Bay Cave Passage based on a minimum radiocarbon age from core 13; C) deposition of carbonate sand during anthropogenic infilling of Eve's Pond in the mid-twentieth century, and D) recovery of the cave benthos following the infilling event. to the modern sediment currently infilling deeper areas in Harrington Sound. Sediment in Harrington Sound is characterized by silt- and clay-sized biogenic debris derived from the biogenic breakdown of algae and the action of boring marine organisms on rocks and other organisms (Thorstenson \& Mackenzie, 1974). It is likely that some of this finedgrained sediment was transported into Green Bay Cave through tidal currents, which would explain the general attenuation of sediment thickness with increasing distance away from Green Bay. The anomalous sand layer, however, was characterized by a mean grain size of $230 \mu \mathrm{m}$ (medium sand), which is similar to the coarser-grained sediment found in shallow lagoons and reefs surrounding most of Bermuda (62 to 4,000 $\mu \mathrm{m}$ ) (Chave, 1962; Garrett et al., 1971). Additionally, the anomalous sand layer was unlike any other sediments found throughout the Holocene history in Green Bay Cave (van Hengstum et al., 2011), thus indicating that it is not a storm layer either. The most likely source of the sand layer is from a shallow lagoon, not Harrington Sound.

The dominant benthic foraminifera from the anomalous sand layers in cores 13 and 17 are consistent with the benthic foraminifera that are found in Bermudian shallow lagoons. In general, the diversity of foraminifera before and after the sand layer was identical, with a Shannon Diversity Index of 3.22, whereas foraminifera diversity was lower in the anomalous sand layer $(H=2.5)$. The Impact Assemblage was dominated by 40\% Quinqueloculina spp., a taxonomic group that does not achieve that level of dominance during the entire Holocene history of Green Bay Cave (van Hengstum \& Scott, 2012). Outside of marine cave environments, Quinqueloculina is a diverse and abundant genus in tropical and subtropical carbonate lagoons in Bermuda (Carman, 1933; Javaux \& Scott, 2003) and elsewhere (Bicchi et al., 2002). Taphonomically, foraminifera genera with robust tests, such as Quinqueloculina, Pyrgo, or Archaias (a taxon with photosymbionts), have a better preservation potential and thus preferentially become incorporated into the sand deposits of shallow, marine carbonate habitats (Martin \& Wright, 1988). The Impact Assemblage also contained a taxa that is common on the reefs and lagoons in Bermuda (e.g., Peneroplis sp.; Javaux \& Scott, 2003), as well a decrease or complete absence of stygophiles (e.g., Spirophthalmidium emaciatum, Sigmoilina tenuis; Javaux \& Scott, 2003; van Hengstum \& Scott, 2011). As such, the lower diversity Impact Assemblage is taphonomically consistent with sediment that was sourced from an adjacent carbonate lagoon.

From a perspective of sedimentary processes, it appears that coarse sand deposition in the cave passage was influenced by the location of boulders from ceiling collapse events (boulder breccias). There are two locations where boulders currently create a noticeable decrease in the depth of the cave passage between Harrington Sound and the 
Letterbox (Figs. 2B, 9). Spatially, the anomalous sand deposit was best preserved in the deeper bathymetry just seaward of the Letterbox, but sediment-probing efforts also documented this deposit mantling the base of boulders elsewhere. During the infilling of Eve's Pond, it is likely that the finer grained particles were preferentially transported out of the cave system (i.e., silts and clays) consistent with eyewitness reports, while coarser grained particles preferentially accumulated near the Letter Box. In addition, core 13 sampled a $4 \mathrm{~cm}$ thick organic matter deposit that was not present in core 17 . This layer perhaps represents the settling out of lower-density and coarser-grained organic matter particles, such as partially decomposed sea grass fragments from outside the cave, after the infilling event. Although the velocity of tidal currents is very low near the Letter Box, tidal currents may still have been sufficient for transporting and reworking lower density organic matter particles into discontinuous deposits.

These results imply that prior to any colonial anthropogenic disturbance, there was most likely a cave passage connecting Eve's Pond to Green Bay Cave. The size, and distance to, such a conduit from the sample locations remains unknown. Given that Bermudian and Mexican anchialine sinkholes, cenotes, or ponds can enable non-marine sediment and microfossils to be transported to interconnected anchialine caves (van Hengstum et al., 2011; van Hengstum \& Scott, 2012), the lack of non-marine sediment and microfossils in core 13 suggest that either (i) the original cave opening into Eve's Pond was not proximal to the Letter Box sampling area, (ii) sediment production in Eve's Pond was insufficient to promote deposition of a discrete stratigraphic deposit in the subsurface, or (iii) the geometry of the cave passage prevented sediment transport to the sampling location (Collins et al., 2015). Previously, the recovery of abundant tests of symbiotic-bearing benthic foraminifera at the terminus of the North Shore Passage led van Hengstum \& Scott (2011) to speculate that the North Shore Passage once opened into the adjunct open water lagoon (see green arrow on Fig. 2A). The knowledge of the opening in Eve's Pond brings the number of known previous 'karst windows' into Green Bay Cave to four. There are several other passages near the Letter Box that remain unsurveyed, and some that divers suspect are filled to the ceiling with poorly-consolidated coarse grained carbonate sediment. Additional exploration and survey is needed to map these passages that may have originally been connected to Eve's Pond, and document their physical condition. It remains challenging to envisage how the original passage from Eve's Pond into Green Bay Cave could be re-breached without more accurate survey data, or causing further harm to adjacent passages that remain pristine.

\section{Recovery of the cave passage}

Since the mid-20th century infilling event, carbonate mud deposition has resumed and benthic foraminifera have re-colonized the dredge spoils that are similar to passages elsewhere that are well-circulated with seawater (Fig. 9D). The infilling event functioned as a natural 'colonization experiment', since multiple taxa were likely introduced into Green Bay Cave from the dredge spoils (e.g., Cibicides), but these foreign taxa did not become established and change the composition of the benthic foraminiferal community. In contrast, there was a near immediate reversion back to an eclectic community of taxa more commonly known from the deep-sea and marine carbonate lagoons, and taxa that thrive in oxygenated marine caves (e.g., $S$. emaciatum and $S$. tenuis). Although this impact event appears to be primarily a physical disturbance, these results do suggest that some benthic fauna of anchialine ecosystems may offer some resiliency to anthropogenic activity. However, the impact of this infilling event on the endemic pelagic crustacean fauna in the cave remains unknown.

It is possible that the disturbed area was rapidly recolonized by foraminiferal propagules (very young juveniles) transported by tidal currents through the cave. In addition to self-locomotion and passive transport as test suspended particles, propagule dispersal is one of many mechanisms in which benthic foraminifera colonize new habitat (Alve \& Goldstein, 2003, 2014). While self-locomotion and passive entrainment have been shown to be efficient over short distances, propagules are light, easily transported, and more prone to travel longer distances (Alve, 1999; Alve \& Goldstein, 2002).

The results further indicate that the benthic foraminifera form unique communities in the submarine cave environment relative to other coastal environments. Benthic foraminiferal assemblages in other Bermudian coastal environments are dominated by specific species, for example, mangroves are dominated by Helenina anderseni and Trichohyalus aguayoi, while lagoons and reefs are dominated by miliolids (Javaux \& Scott, 2003; Carmen, 1933). In contrast, the marine caves contain taxa that are more commonly known from deeper marine habitats (e.g., Globocassidulina subglobosa: 6\%, Melonis barleeanum: $~ 5 \%$, Spiropthalmidium emaciatum: $~ 7 \%$; Table 2). For example, sediment samples from the northern margin of Little Bahama Bank show negligible abundance of Globocassidulina subglobosa at depths $<200 \mathrm{~m}$, however, as depth increases $(>900 \mathrm{~m})$, their abundance increases to approximately $40 \%$ of the total deep-water assemblage (Martin, 1988). In addition, the abundant stygophile Spirophthalmidium emaciatum has only been reported elsewhere from deep-sea habitats in the Mediterranean, high latitude North Atlantic, and southeast Indian Ocean (Corliss, 1979; Hermelin \& Scott, 1985; Abu-Zied et al., 2008). Collectively, the entire submarine cave assemblage is a unique grouping of taxa that has been documented previously from other well-circulated passages of Green Bay Cave (van Hengstum \& Scott, 2011). Therefore, the benthic foraminiferal assemblage observed in the modern carbonate mud of Green Bay Cave forms a unique meiofaunal community that is specifically adapted to life in an underwater cave.

Outside of Bermuda, global cave environments face increasing pressure from coastal urbanization 
and tourism. In some cases, landowners consider altering cave geometries by increasing karst windows to increase tourism revenue, or infilling passages to accommodate infrastructure projects. The results from Green Bay Cave serve as an observational record that sediment infilling can impact cave passages located some distance away from the infilling site. Conversely, addition of new karst windows will potentially offer a new vector for sediment and contaminants to more easily reach otherwise pristine subterranean habits. And, our understanding of how the sudden opening-or-closing of karst windows by humans impacts the pelagic cave fauna remains unknown (e.g., endemic crustaceans).

\section{CONCLUSIONS}

- Deposition in Green Bay Cave, at least over the last $\sim 1,900$ years, has been dominated by carbonate mud. Benthic foraminiferal assemblages over this time have responded to increased oxygenation at the sediment-water interface. This change in oxygenation was most likely related to increased circulation of well-oxygenated seawater from Harrington Sound into the relatively oxygen-poor saline groundwater flooding the cave habitats.

- A spatially-discontinuous carbonate sand deposit with lagoon foraminifera has recently accumulated in some areas of Green Bay Cave (i.e., proximal to the Letter Box area). This discrete sedimentary deposit is anomalous with respect the Holocene depositional history of the cave.

- Based on benthic foraminifera and sediment texture, this anomalous carbonate sand deposit was most likely emplaced during the infilling of Eve's Pond in the mid-20th century using dredge spoils derived from Flatt's Inlet between Harrington Sound and the North Shore Lagoon. The implications are that Green Bay Cave previously had an additional cave entrance (karst window) that is now filled in.

- After the infilling event, benthic foraminifera reverted to an assemblage that is characteristic of well oxygenated submarine cave environments in Bermuda, including the prevalence of stygophiles (e.g., Spirophthalmidium emaciatum, Sigmoilina tenuis). This further indicates that Bermudian marine caves host unique benthic foraminiferal communities that differ from those found in other marine environments (lagoons, marshes, fore reef slope, etc.).

- Although this impact event was primarily a physical disturbance, these results do suggest that benthic fauna of anchialine ecosystems may offer some resiliency to anthropogenic activity. However, the impact of this infilling event on the endemic pelagic crustacean fauna remains unknown.

\section{ACKNOWLEDGEMENTS}

This research was funded by the John W. Hess Student Research Grant in Karst Research Studies from the Geologic Society of America (JNC, EAR-
1354519), the Bermuda Zoological Society (JNC), the Bermuda Aquarium, Museum \& Zoo (JNC), Texas A\&M University at Galveston Marine Biology student grants (JNC), and NSF grants OCE-151955 (PvH) and OCE-1356509 (PvH). Three anonymous reviewers are thanked for providing comments to improve an earlier version of this paper. This is Contribution \#259, Bermuda Biodiversity Project (BBP), Bermuda Aquarium, Museum and Zoo, Department of Environment and Natural Resources.

\section{REFERENCES}

Alve E., 1999 - Colonization of new habitats by benthic foraminifera: a review. Earth-Science Reviews, 46 (1): 167-185.

https://doi.org/10.1016/S0012-8252(99)00016-1

Alve E. \& Goldstein S.T., 2002 - Resting stage in benthic foraminiferal propagules: a key feature for dispersal? Evidence from two shallow-water species. Journal of Micropalaeontology, 21 (1): 95-96.

https://doi.org/10.1144/jm.21.1.95

Alve E. \& Goldstein S.T., 2003 - Propagule transport as a key method of dispersal in benthic foraminifera (Protista). Limnology and Oceanography, 48 (6): 21632170. https://doi.org/10.4319/10.2003.48.6.2163

Alve E. \& Goldstein S.T., 2014 - The propagule method as an experimental tool in foraminiferal ecology. Approaches to study living foraminifera. Springer, Berlin, p. 1-12.

Angel M.V. \& Iliffe T.M., 1987 - Spelaeoecia bermudensis, new genus, new species, a halocyprid ostracod from marine caves in Bermuda. Journal of Crustacean Biology, 7 (3): 541-553.

https://doi.org/10.2307/1548303

Bermúdez P.J., 1949 - Tertiary smaller foraminifera of the Dominican Republic. Cushman Laboratory for Foraminiferal Research, Special Publication 25, Sharon, Massachusetts, $322 \mathrm{p}$.

Bernhard J.M. \& Gupta B.K.S., 1999 - Foraminifera of oxygen-depleted environments. Modern foraminifera. Springer, Berlin, p. 201-216.

Bicchi E., Debenay J.-P. \& Pages J., 2002 - Relationship between benthic foraminiferal assemblages and environmental factors in atoll lagoons of the central Tuamotu Archipelago (French Polynesia). Coral Reefs, 21 (3): 275-290. https://doi.org/10.1007/s00338-002-0245-6

Boltovskoy E., Scott D.B. \& Medioli F., 1991 Morphological variations of benthic foraminiferal tests in response to changes in ecological parameters: a review. Journal of Paleontology, 65 (2): 175-185. https://doi.org/10.1017/S0022336000020394

Bowman T.E. \& Iliffe T.M., 1985 - Mictocaris halope, a new unusual peracaridan crustacean from marine caves on Bermuda. Journal of Crustacean Biology, 5 (1): 58-73. https://doi.org/10.2307/1548220

Carman K.W., 1933 - The shallow-water foraminifera of Bermuda. Unpublished PhD thesis, Massachusetts Institute of Technology.

Chave K.E., 1962 - Factors influencing the mineralogy of carbonate sediments. Limnology and Oceanography, 7 (2): 218-223.

https://doi.org/10.4319/1o.1962.7.2.0218

Chevaldonné P. \& Lejeusne C., 2003 - Regional warminginduced species shift in north-west Mediterranean marine caves. Ecology Letters, 6 (4): 371-379. https://doi.org/10.1046/j.1461-0248.2003.00439.x 
Collins S., Reinhardt E., Werner C., Le Maillot C., Devos F. \& Rissolo D., 2015 - Late Holocene mangrove development and onset of sedimentation in the Yax Chen cave system (Ox Bel Ha) Yucatan, Mexico: Implications for using cave sediments as a sea-level indicator. Palaeogeography, Palaeoclimatology, Palaeoecology, 438: $124-134$.

https://doi.org/10.1016/j.palaeo.2015.07.042

Corliss, B. H., 1979- Taxonomy of recent deep-sea benthonic foraminifera from the southeast Indian Ocean. Micropaleontology, 25 (1): 1-19.

https://doi.org/10.2307/1485207

Dissard D., Nehrke G., Reichart G.J. \& Bijma J., 2010 - The impact of salinity on the $\mathrm{Mg} / \mathrm{Ca}$ and $\mathrm{Sr} / \mathrm{Ca}$ ratio in the benthic foraminifera Ammonia tepida: Results from culture experiments. Geochimica et Cosmochimica Acta, 74 (3): 928-940.

https://doi.org/10.1016/j.gca.2009.10.040

Fornós J.J., Ginés J., Gràcia F., Merino Juncadella A., Gómez-Pujol L. \& Bover P., 2014 - Cave deposits and sedimentary processes in Cova des Pas de Vallgornera (Mallorca, Western Mediterranean). International Journal of Speleology, 43 (2): 159-174.

https://doi.org/10.5038/1827-806X.43.2.5

Garrett P., Smith D.L., Wilson A.O. \& Patriquin D., 1971 - Physiography, ecology, and sediments of two Bermuda patch reefs. The Journal of Geology, 79 (6): 647-668. https://doi.org/10.1086/627696

Gibbons D.A., 2003 - An environmental assessment of Bermuda's caves. Unpublished thesis, Texas A\&M University.

Gooday A.J., Levin L.A., Linke P. \& Heeger T., 1992 - The role of benthic foraminifera in deep-sea food webs and carbon cycling. Deep-sea food chains and the global carbon cycle. Springer Netherlands, p. 63-91. https://doi.org/10.1007/978-94-011-2452-2_5

Gupta B.K.S. \& Machain-Castillo M.L., 1993 Benthic foraminifera in oxygen-poor habitats. Marine Micropaleontology, 20 (3-4): 183-201. https://doi.org/10.1016/0377-8398(93)90032-S

Hart C. \& Manning R., 1986 - Two new shrimps (Procarididae and Agostocarididae, new family) from marine caves of the western North Atlantic. Journal of Crustacean Biology, 6 (3): 408-416. https://doi.org/10.2307/1548181

Hayami I., 1993 - Submarine cave Bivalvia from the Ryukyu Islands: systematics and evolutionary significance. Bulletin University Museum, University of Tokyo, 35: 1-133.

Heiri O., Lotter A.F. \& Lemcke G., 2001 - Loss on ignition as a method for estimating organic and carbonate content in sediments: reproducibility and comparability of results. Journal of paleolimnology, 25 (1): 101-110. https://doi.org/10.1023/A:1008119611481

Hermelin J.O.R. \& Scott D.B., 1985 - Recent benthic foraminifera from the central North Atlantic. Micropaleontology, 31: 199-220.

https://doi.org/10.2307/1485542

Iliffe T.M., Hart C. \& Manning R.B., 1983 - Biogeography and the caves of Bermuda. Nature, 302: 141-142. https://doi.org/10.1038/302141a0

Iliffe T.M., Wilkens H., Parzefall J. \& Williams D., 1984 - Marine lava cave fauna: composition, biogeography, and origins. Science, 225: 309-311.

https://doi.org/10.1126/science.225.4659.309

Javaux E.J. \& Scott D.B., 2003 - Illustration of modern benthic foraminifera from Bermuda and remarks on distribution in other subtropical/tropical areas. Palaeontologia Electronica, 6 (4): 29 p.
Jorissen F.J., 1999 - Benthic foraminiferal microhabitats below the sediment-water interface. Modern foraminifera. Springer, p. 161-179.

Kaiho K., 1994 - Benthic foraminiferal dissolved-oxygen index and dissolved-oxygen levels in the modern ocean. Geology, 22 (8): 719-722.

https://doi.org/10.1130/0091-7613(1994)022 $\leq 0719$ :BFDOIA $>2.3 . \mathrm{CO} ; 2$

Kano Y. \& Kase T., 2008 - Diversity and distributions of the submarine-cave Neritiliidae in the Indo-Pacific (Gastropoda: Neritimorpha). Organisms Diversity \& Evolution, 8 (1): 22-43.

https://doi.org/10.1016/j.ode.2006.09.003

Kase T. \& Hayami I., 1992 - Unique submarine cave mollusc fauna: composition, origin and adaptation. Journal of Molluscan Studies, 58 (4): 446-449. https://doi.org/10.1093/mollus/58.4.446

Kitamura A., Hiramoto M., Kase T., Yamamoto N., Amemiya M. \& Ohashi S., 2007 - Changes in cavernicolous bivalve assemblages and environments within a submarine cave in the Okinawa Islands during the last 5,000 years. Paleontological Research, 11 (2): 163-182.

https://doi.org/10.2517/1342-8144(2007)11 [163:CICBAA]2.0.CO;2

Kløve B., Balderacchi M., Gemitzi A., Hendry S., Kværner J., Muotka T. \& Preda E., 2014 - Protection of groundwater dependent ecosystems: current policies and future management options. Water Policy, 16 (6): 1070-1086.

https://doi.org/10.2166/wp.2014.014

Kornicker L.S. \& Iliffe T.M., 1998 - Myodocopid Ostracoda (Halocypridina, Cladocopina) from anchialine caves in the Bahamas, Canary Islands, and Mexico. Smithsonian Contributions to Zoology, 599: 1-93.

https://doi.org/10.5479/si.00810282.599

Land L.S., Mackenzie F.T. \& Gould S.J., 1967 Pleistocene history of Bermuda. Geological Society of America Bulletin, 78 (8): 993-1006. https://doi.org/10.1130/0016-7606(1967)78 [993:PHOB]2.0.CO;2

Lea D.W., Pak D.K., Peterson L.C. \& Hughen K.A., 2003 - Synchroneity of tropical and high-latitude Atlantic temperatures over the last glacial termination. Science, 301: 1361-1364.

https://doi.org/10.1126/science. 1088470

Legendre P. \& Legendre L.F., 1998 - Numerical ecology. Developments in environmental modeling, 20, Elsevier, Amsterdam, $853 \mathrm{p}$.

Leutenegger S. \& Hansen H., 1979 - Ultrastructural and radiotracer studies of pore function in foraminifera. Marine Biology, 54 (1): 11-16. https://doi.org/10.1007/BF00387046

Loeblich A.R. Jr. \& Tappan H., 1987 - Foraminiferal genera and their classification. Van Nostrand Reinhold Company, New York, 2 vols., 970 p.

Maddocks R.F. \& Iliffe T.M., 1986 - Podocopid ostracoda of Bermudian caves. Stygologia, 2 (1-2): 26-76.

Martin R.E., 1988 - Benthic foraminiferal zonation in deep-water carbonate platform margin environments, Northern Little Bahama Banks. Journal of Paleontology, 62 (1): 1-8.

https://doi.org/10.1017/S0022336000017935

Martin R.E. \& Wright R.C., 1988 - Information loss in the transition from life to death assemblages of foraminifera in back reef environments, Key Largo, Florida. Journal of Paleontology, 62 (3): 399-410. https://doi.org/10.1017/S002233600001831X 
Martin P.A., Lea D.W., Rosenthal Y., Shackleton N.J., Sarnthein M. \& Papenfuss T., 2002 - Quaternary deep sea temperature histories derived from benthic foraminiferal $\mathrm{Mg} / \mathrm{Ca}$. Earth and Planetary Science Letters, 198 (1): 193-209.

https://doi.org/10.1016/S0012-821X(02)00472-7

Metcalfe C.D., Beddows P.A., Bouchot G.G., Metcalfe T.L., Li H. \& Van Lavieren H., 2011 - Contaminants in the coastal karst aquifer system along the Caribbean coast of the Yucatan Peninsula, Mexico. Environmental pollution, 159 (4): 991-997.

https://doi.org/10.1016/j.envpol.2010.11.031

Miller T.E., 1996 - Geologic and hydrologic controls on karst and cave development in Belize. Journal of Cave and Karst Studies, 58 (2): 100-120.

Moolenbeek R., Faber M. \& Iliffe T.M., 1989 - Two new species of the genus Caecum (Gastropoda) from marine caves on Bermuda. Studies in honour of Dr. Pieter Wagenaar Hummelinch. Foundation for Scientific Research in Surinam and the Netherlands Antilles, Amsterdam, 123: 209-216.

Moritsch M.M., Pakes M.J. \& Lindberg D.R., 2014 - How might sea level change affect arthropod biodiversity in anchialine caves: a comparison of Remipedia and Atyidae taxa (Arthropoda: Altocrustacea). Organisms Diversity \& Evolution, 14 (2): 225-235. https://doi.org/10.1007/s13127-014-0167-5

Murray J.W., 2001 - The niche of benthic foraminifera, critical thresholds and proxies. Marine Micropaleontology, 41 (1): 1-7. https://doi.org/10.1016/S0377-8398(00)00057-8

Mylroie J.E., Carew J.L. \& Vacher H., 1995 - Karst development in the Bahamas and Bermuda. In: Curran H.A. \& White B. (Eds.), Terrestrial and shallow marine geology of The Bahamas and Bermuda. Geological Society of America Special Paper, 300: 251-267. https://doi.org/10.1130/0-8137-2300-0.251

Mylroie J.R. \& Mylroie J.E., 2007 - Development of the carbonate island karst model. Journal of Cave and Karst Studies, 69 (1): 59-75.

Nevill J.C., Hancock P.J., Murray B.R., Ponder W.F., Humphreys W.F., Phillips M.L. \& Groom P.K., 2010 Groundwater-dependent ecosystems and the dangers of groundwater overdraft: a review and an Australian perspective. Pacific Conservation Biology, 16 (3): 187208. https://doi.org/10.1071/PC100187

Parravicini V., Guidetti P., Morri C., Montefalcone M., Donato M. \& Bianchi C.N., 2010 - Consequences of sea water temperature anomalies on a Mediterranean submarine cave ecosystem. Estuarine, Coastal and Shelf Science, 86 (2): 276-282.

https://doi.org/10.1016/j.ecss.2009.11.004

Petersen J., Riedel B., Barras C., Pays O., Guihéneuf A., Mabilleau G., Schweizer M., Meysman F.J. \& Jorissen F.J., 2016 - Improved methodology for measuring pore patterns in the benthic foraminiferal genus Ammonia. Marine Micropaleontology, 128: 1-13.

https://doi.org/10.1016/j.marmicro.2016.08.001

Radolović M., Bakran-Petricioli T., Petricioli D., Surić M. $\&$ Perica D., 2015 - Biological response to geochemical and hydrological processes in a shallow submarine cave. Mediterranean Marine Science, 16 (2): 305-324. https://doi.org/10.12681/mms.1146

Reimer P.J., Bard E., Bayliss A., Beck J.W., Blackwell P.G., Bronk Ramsey C., Buck C.E., Cheng H., Edwards R.L. \& Friedrich M., 2013 - IntCal13 and Marine13 radiocarbon age calibration curves $0-50,000$ years cal $B P$. Radiocarbon, 55 (4): 1869-1887.

https://doi.org/10.2458/azu_js_rc.55.16947
Sarbu S.M., Kane T.C. \& Kinkle B.K., 1996 - A chemoautotrophically based cave ecosystem. Science, 272: 1953-1954.

https://doi.org/10.1126/science.272.5270.1953

Savage A.J., 1901 - Islands of Bermuda. Six inches to One Statue Mile, Southampton, Ordnance Survey Office.

Scott D.B. \& Hermelin J., 1993 - A device for precision splitting of micropaleontological samples in liquid suspension. Journal of Paleontology, 67 (1): 151-154. https://doi.org/10.1017/S0022336000021302

Sperazza M., Moore J.N. \& Hendrix M.S., 2004 - Highresolution particle size analysis of naturally occurring very fine-grained sediment through laser diffractometry. Journal of Sedimentary Research, 74 (5): 736-743. https://doi.org/10.1306/031104740736

Thorstenson D.C. \& Mackenzie F.T., 1974 - Time variability of pore water chemistry in recent carbonate sediments, Devil's Hole, Harrington Sound, Bermuda. Geochimica et Cosmochimica Acta, 38 (1): 1-19. https://doi.org/10.1016/0016-7037(74)90192-6

Vacher H.L., 1988 - Dupuit-Ghyben-Herzberg analysis of strip-island lenses. Geological Society of America Bulletin, 100 (4): 580-591.

https://doi.org/10.1130/0016-7606(1988)100 $<0580$ :DGHAOS $>2.3 . \mathrm{CO} ; 2$

van Hengstum P.J. \& Scott D.B., 2011 - Ecology of foraminifera and habitat variability in an underwater cave: distinguishing anchialine versus submarine cave environments. Journal of Foraminiferal Research, 41 (3): 201-229. https://doi.org/10.2113/gsjfr.41.3.201

van Hengstum P.J. \& Scott D.B., 2012 - Sea-level rise and coastal circulation controlled Holocene groundwater development in Bermuda and caused a meteoric lens to collapse 1600 years ago. Marine Micropaleontology, 90-91: 29-43.

https://doi.org/10.1016/j.marmicro.2012.02.007

van Hengstum P.J., Scott D.B. \& Javaux E.J., 2009b - Foraminifera in elevated Bermudian caves provide further evidence for $+21 \mathrm{~m}$ eustatic sea level during Marine Isotope Stage 11. Quaternary Science Reviews, 28 (19-20): 1850-1860.

https://doi.org/10.1016/j.quascirev.2009.05.017

van Hengstum P.J., Reinhardt E.G., Beddows P.A. \& Gabriel J.J., 2010 - Linkages between Holocene paleoclimate and paleohydrogeology preserved in a Yucatan underwater cave. Quaternary Science Reviews, 29 (19-20): 2788-2798.

https://doi.org/10.1016/j.quascirev.2010.06.034

van Hengstum P.J., Richards D.A., Onac B.P. \& Dorale J.A., 2015a - Coastal caves and sinkholes. In: Shennan I., Long A.J. \& Horton B.P. (Eds.), Handbook of sealevel research. John Wiley \& Sons, p. 83-103.

https://doi.org/10.1002/9781118452547.ch6

van Hengstum P.J., Scott D.B., Gröcke D.R. \& Charette M.A., 2011 - Sea level controls sedimentation and environments in coastal caves and sinkholes. Marine Geology, 286 (1-4): 35-50.

https://doi.org/10.1016/j.margeo.2011.05.004

van Hengstum P.J., Reinhardt E.G., Beddows P.A., Schwarcz H.P. \& Gabriel J.J., 2009a - Foraminifera and testate amoebae (thecamoebians) in an anchialine cave: surface distributions from Aktun $\mathrm{Ha}$ (Carwash) cave system, Mexico. Limnology and Oceanography, 54 (1): 391-396. https://doi.org/10.4319/10.2009.54.1.0391 van Hengstum P.J., Donnelly J.P., Kingston A.W., Williams B.E., Scott D.B., Reinhardt E.G., Little S.N. \& Patterson W.P., 2015b - Low-frequency storminess signal at Bermuda linked to cooling events in the North Atlantic region. Paleoceanography, 30: 52-76. https://doi.org/10.1002/2014PA002662 
Vollbrecht R.D., 1996 - Postglazialer Anstieg des Meeresspiegels, paläoklima und hydrographie, aufgezeichnet in sedimenten der Bermuda inshore waters. Ph. D. Dissertation, Universität Göttingen, Göttingen, Germany.
Waelbroeck C., Labeyrie L., Michel E., Duplessy J.C., McManus J., Lambeck K., Balbon E. \& Labracherie M., 2002 - Sea-level and deep water temperature changes derived from benthic foraminifera isotopic records. Quaternary Science Reviews, 21 (1): 295-305. https://doi.org/10.1016/S0277-3791(01)00101-9 